\title{
A DOMAIN DECOMPOSITION ANALYSIS FOR A TWO-SCALE LINEAR TRANSPORT PROBLEM
}

\author{
FranÇOis Golse $^{1}$, Shi Jin ${ }^{2}$ And C. David Levermore ${ }^{3}$
}

\begin{abstract}
We present a domain decomposition theory on an interface problem for the linear transport equation between a diffusive and a non-diffusive region. To leading order, i.e. up to an error of the order of the mean free path in the diffusive region, the solution in the non-diffusive region is independent of the density in the diffusive region. However, the diffusive and the non-diffusive regions are coupled at the interface at the next order of approximation. In particular, our algorithm avoids iterating the diffusion and transport solutions as is done in most other methods - see for example Bal-Maday (2002). Our analysis is based instead on an accurate description of the boundary layer at the interface matching the phase-space density of particles leaving the non-diffusive region to the bulk density that solves the diffusion equation.
\end{abstract}

Mathematics Subject Classification. 65N55, 82B40, 82B80, 82C40, 82C70, 76R50.

Received: February 17, 2003.

\section{THE INTERFACE PROBLEM}

Consider the steady, linear transport equation with isotropic scattering and slab geometry:

$$
\begin{aligned}
\mu \partial_{x} \Psi(x, \mu)+\sigma(x) \Psi(x, \mu) & =\sigma(x) c(x) \bar{\Psi}(x), \\
\text { where } \bar{\Psi}(x) & =\frac{1}{2} \int_{-1}^{1} \Psi(x, \mu) \mathrm{d} \mu .
\end{aligned}
$$

The phase space density $\Psi$ is defined so that $\Psi(x, \mu) \frac{1}{2} \mathrm{~d} \mu \mathrm{d} x$ is the number of particles (e.g. neutrons) located inside an interval of width $\mathrm{d} x$ centered at $x$, moving in a direction whose angle $\theta$ with the $x$ axis is such that $\mu=\cos \theta$ belongs to an interval of width $\mathrm{d} \mu$ centered at $\mu$. The function $\sigma(x)>0$ is the scattering cross-section at position $x$, while $c(x)>0$ is the average number of emitted particles per collision at $x$. Below we assume that $0<c(x) \leq 1$. When $c=1$ the material is purely scattering; when $c<1$ there exist absorbing collisions. The transport equation (1.1) is posed for $x \in\left(x_{L}, x_{R}\right)$ and $\mu \in[-1,1]$, supplemented with boundary conditions at $x_{L}$

\footnotetext{
Keywords and phrases. Domain decomposition, transport equation, diffusion approximation, kinetic-fluid coupling.

1 Institut Universitaire de France, Département de Mathématiques et Applications, École Normale Supérieure Paris, 45 rue d'Ulm, 75230 Paris Cedex 05, France. e-mail: golse@dma.ens.fr

2 Department of Mathematics, University of Wisconsin-Madison, Madison, Wisconsin 53706, USA. e-mail: jin@math.wisc.edu

3 Department of Mathematics, Institute of Physical Sciences and Technology, University of Maryland, College Park, Maryland 20742, USA. e-mail: lvrmr@math.umd.edu

(C) EDP Sciences, SMAI 2003
} 
and $x_{R}$. Perhaps the simplest example of boundary conditions for (1.1) consists in prescribing the phase-space density of particles entering the domain $\left(x_{L}, x_{R}\right)$ at $x_{L}$ and $x_{R}$ :

$$
\begin{aligned}
\Psi\left(x_{L}, \mu\right) & =F_{L}(\mu), & & \text { for } \mu \in(0,1], \\
\Psi\left(x_{R},-\mu\right) & =F_{R}(\mu), & & \text { for } \mu \in(0,1] .
\end{aligned}
$$

More general boundary conditions can also be analyzed by the methods of the present paper. For more details on the physical meaning of $(1.1,1.2)$, the interested reader is referred to chapter XXI of [7]. Our purpose is to analyze the solution of $(1.1,1.2)$ in the case where the order of magnitude of the scattering cross-section $\sigma$ varies considerably over the domain $\left(x_{L}, x_{R}\right)$. Such situations are frequently encountered in most applications of transport theory where the background medium is often made of (very) different materials. Specifically, we consider the case of two different materials with an interface located at $x_{M} \in\left(x_{L}, x_{R}\right)$. At $x_{M}$, the scattering cross-section $\sigma$ and emission rate $c(x)$ are assumed to be discontinuous; they are given as follows in terms of a small parameter $\epsilon$ :

$$
\begin{aligned}
& \sigma(x)=1, \quad \text { and } \quad 0<c_{*} \leq c(x) \leq c^{*}<1, \quad \text { for } x \in\left(x_{L}, x_{M}\right) \text {, } \\
& \sigma(x)=\epsilon^{-1}, \quad \text { and } \quad c(x)=1-\epsilon^{2} \gamma(x), \quad \text { for } x \in\left(x_{M}, x_{R}\right) \text {. }
\end{aligned}
$$

The mathematical analysis of our coupling algorithm will be done under the following assumptions:

$$
0<\gamma_{*} \leq \gamma(x) \leq \gamma^{*}, \quad \text { for each } x \in\left(x_{M}, x_{R}\right)
$$

for some constants $\gamma_{*}$ and $\gamma^{*}$; we shall also restrict our attention to $\epsilon$ 's such that

$$
0<\epsilon<\epsilon^{*}, \quad \text { where } \epsilon^{*}<1 / \gamma^{*} .
$$

Notice that these assumptions exclude the case where $c$ takes the value 1: in particular, the case of a purely scattering, low $\sigma$ medium is excluded by the assumption (1.3). However, this is only for mathematical convenience, and our algorithm also works in that case: we shall return to this after the statement of our main results, Theorems 3.1 and 3.2 .

The small parameter $\epsilon$ is the ratio of the mean free path (the average distance a particle travels between two consecutive collisions with the background medium) to the size of the domain $x_{R}-x_{M}$. Changing the space variable $x$ in (1.1) into

$$
\begin{aligned}
\quad x_{M}-\int_{x}^{x_{M}} \sigma(z) \mathrm{d} z & \text { for } x \in\left(x_{L}, x_{M}\right), \\
\text { and } x_{M}+\int_{x_{M}}^{x} \sigma(z) \mathrm{d} z & \text { for } x \in\left(x_{M}, x_{R}\right),
\end{aligned}
$$

one sees that there is no loss of generality in assuming $\sigma$ to be piecewise constant as in (1.3). (In the context of radiative transfer, the new space variables so defined are referred to as the "optical thickness": see Chap. I, Sect. 7 in [6].) Since the mean-free path is small in the region $\left(x_{M}, x_{R}\right)$, we expect that the solution $\Psi_{\epsilon}$ is isotropic (i.e. a function of $x$ alone) to leading order and governed by the diffusion approximation of (1.1): see Section 4.1 below. Hence the domain $\left(x_{M}, x_{R}\right)$ is referred to as "the diffusive region". The smallness of the mean free path in $\left(x_{M}, x_{R}\right)$ makes it very costly to solve the transport equation accurately there. Solving the diffusion equation on the other hand is much more efficient. By contrast, in the domain $\left(x_{L}, x_{M}\right)$ one must retain the $\mu$ dependence in the solution $\Psi_{\epsilon}$ and solve the transport equation for $\Psi_{\epsilon}$ in that domain, which will be therefore referred to as "the transport region". Domain decomposition methods matching kinetic and hydrodynamic or diffusion models have received a lot of attention in the past 15 years. Some of the ideas in the present work can be found in [10]; other methods have been proposed in $[1,4,8,9,13,17,18,20,21,24-26]$. In the notations of Figure 1, solving the problem $(1.1,1.2)$ with coefficients given by $(1.3)$ reduces to finding good 


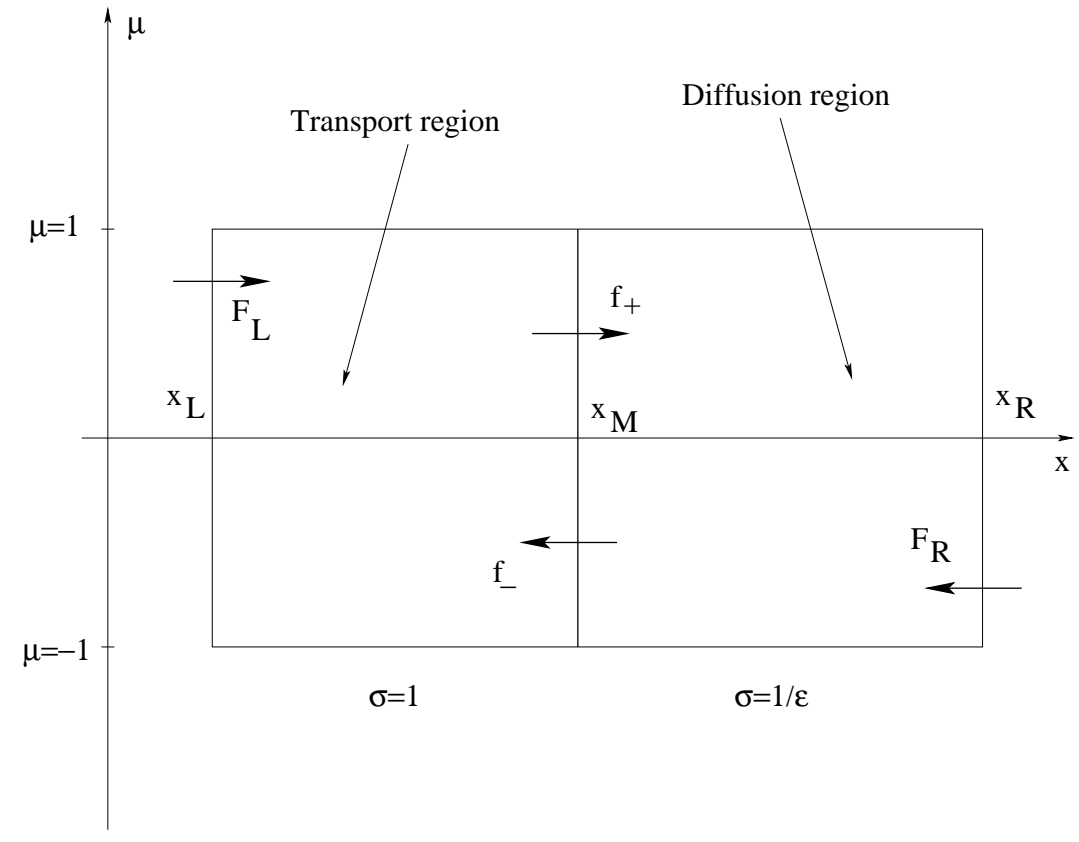

Figure 1. The interface problem.

approximations of the angle distributions of particles crossing the interface in the direction of the transport domain - i.e. $f_{-}$- and in the direction of the diffusive domain - i.e. $f_{+}$. In fact, the solution in the diffusive region depends only on some appropriate angle average of $f_{+}$, so that the most critical task is to evaluate $f_{-}$ accurately. In most of the existing domain decomposition methods for this problem — for instance in $[1,26]$ this is done by an iteration procedure in which the diffusion and the transport equations are solved alternately until convergence of the successive approximants to $f_{-}$and $f_{+}$is reached. The method described in this paper is completely different. Instead, we propose a boundary condition at $x_{M}$ on the transport side which mimics the reflection of particles on an infinitely thick, purely scattering domain. This reflection condition is based on almost explicit computations for the steady transport equation in a half-space filled with a purely scattering material. These computations go back to the work of Wiener and Hopf [27] on the Milne-Schwarzschild problem in astrophysics. This material is recalled in Section 2. Then, classical results on the diffusion approximation of the transport equation show that, by solving the transport equation in the transport region $\left(x_{L}, x_{M}\right)$ with this reflection condition at $x_{M}$, one approximates the restriction to the transport domain of the global solution to within $O(\epsilon)$. Thus, using this reflection condition yields the correct transport and diffusion solutions in one step up to an $O(\epsilon)$ error and in two steps (a prediction-correction algorithm) up to an $O\left(\epsilon^{2}\right)$ error. In particular, this method avoids iterating alternately on the diffusion and transport solutions until convergence of the fluxes at the interface is reached. This simple model can be seen as the prototype in a series of analogous problems, such as, for instance, radiative transfer in multi-material media, gas (or plasma) dynamics in cases involving both a kinetic and a hydrodynamic description etc. We hope that a complete and rigorous treatment of this simple case can give valuable insight on some of the issues listed above.

The domain decomposition method proposed here applies to problems where $\sigma(x)$ is discontinuous. If $\sigma(x)$ varies continuously from $O(1)$ to $O(\epsilon)$, then there is a smooth transition between the transport and the diffusion regions. In such a case, one can use the asymptotic-preserving schemes developed in $[5,16,19]$, where one solves just the transport equation but allows coarse mesh sizes and large time steps (compared with $\epsilon$ ) in the diffusive region. 


\section{Half-Space problems, Chandrasekhar's $H$-Function, REFLECTION ON A SEMI-INFINITE, PURELY SCATTERING MEDIUM}

In this section, we quickly review the mathematical objects involved in the reflection condition at the interface between the transport and the diffusive domains. Consider the half-space transport problem

$$
\mu \partial_{z} \Gamma+\Gamma-\bar{\Gamma}=0, \quad z>0,0<|\mu|<1,
$$

with boundary condition

$$
\Gamma(0, \mu)=G(\mu), \quad 0<\mu<1 .
$$

The theory of existence, uniqueness and asymptotic behavior of this half-space transport problem follows from the work of Wiener and Hopf [27]. It is summarized in the next lemma which can be proved by energy (PDE) methods - this was done originally in [2], with a few technical improvements in [12] (see pp. 1359-1368 there).

Lemma 2.1. For each $G \in L^{2}([0,1], \mu \mathrm{d} \mu)$ there is a unique solution $\Gamma$ in $L^{\infty}\left([0,+\infty) ; L^{2}([-1,1],|\mu| \mathrm{d} \mu)\right)$ to the half-space transport equation (2.1). This solution satisfies the following properties:

- if $G \in L^{\infty}([0,1])$, then $\Gamma \in L^{\infty}([0,+\infty) \times[-1,1])$ and one has

$$
\|\Gamma\|_{L^{\infty}} \leq\|G\|_{L^{\infty}} ;
$$

- it has zero flux

$$
\frac{1}{2} \int_{-1}^{1} \mu \Gamma(z, \mu) \mathrm{d} \mu=0 ;
$$

- there exists a constant $\Gamma_{\infty}$ such that, for each $\gamma \in[0,1)$

$$
\begin{aligned}
\left\|\Gamma(z, \cdot)-\Gamma_{\infty}\right\|_{L^{2}(|\mu| \mathrm{d} \mu)} & =O\left(\mathrm{e}^{-\gamma z}\right), & & \text { and } \\
\left\|\Gamma(z, \cdot)-\Gamma_{\infty}\right\|_{L^{\infty}} & =O\left(\mathrm{e}^{-\gamma z}\right), & & \text { if } G \in L^{\infty}([0,1])
\end{aligned}
$$

as $z \rightarrow+\infty$.

Notice that $(z, \mu) \mapsto z-\mu$ is a solution of $(2.1)$; hence, by Lemma 2.1 , the half-space problem $(2.1,2.2)$ with $G \equiv 0$ has a unique solution $\Gamma_{*}$ such that $(z, \mu) \mapsto \Gamma_{*}(z, \mu)-z$ belongs to $L^{\infty}([0,+\infty) \times[-1,1])$. (In other words, $\Gamma_{*}=\Gamma_{*}^{0}+z-\mu$ where $\Gamma_{*}^{0}$ is the unique bounded solution of $(2.1,2.2)$ with $G(\mu) \equiv \mu$.)

Definition 2.1. The Chandrasekhar $H$-function is defined as

$$
H(\mu)=\frac{1}{\sqrt{3}} \Gamma_{*}(0,-\mu), \quad \mu \in(0,1) .
$$

In particular ${ }^{1}, H \in L^{\infty}([0,1])$.

By Lemma 2.1, the map $G \mapsto \Gamma_{\infty}$ defines a linear functional on $L^{2}([0,1], \mu \mathrm{d} \mu)$; likewise, $\Gamma(0,-\mu)=(\mathcal{R} G)(\mu)$ a.e. on $[0,1]$ where $\mathcal{R}$ is a bounded operator on $L^{2}([0,1], \mu \mathrm{d} \mu)$. The striking fact in the theory of the half-space problem (2.1) is that both this linear functional and the operator $\mathcal{R}$ have remarkably simple expressions in terms of the Chandrasekhar $H$-function.

Lemma 2.2. For each $G \in L^{2}([0,1], \mu \mathrm{d} \mu)$ the unique solution $\Gamma$ of the half-space transport equation (2.1) in $L^{\infty}\left([0,+\infty) ; L^{2}([-1,1],|\mu| \mathrm{d} \mu)\right)$ satisfies the following properties:

- as $z \rightarrow+\infty, \Gamma(z, \cdot)$ converges in $L^{2}([-1,1],|\mu| \mathrm{d} \mu)$ to

$$
\Gamma_{\infty}=\frac{\sqrt{3}}{2} \int_{0}^{1} G(\mu) H(\mu) \mu \mathrm{d} \mu ;
$$

\footnotetext{
${ }^{1}$ One also knows that $\mu \mapsto \mu H(\mu)$ is in $W^{1,1}([0,1])$ - see [12], Theorem C.3 - but this is not needed below.
} 
- for a.e. $\mu \in(0,1]$, the density of particles emerging from the half-space is

$$
\Gamma(0,-\mu)=(\mathcal{R} G)(\mu)=\frac{1}{2} H(\mu) \int_{0}^{1} G\left(\mu^{\prime}\right) \frac{H\left(\mu^{\prime}\right)}{\mu+\mu^{\prime}} \mu^{\prime} \mathrm{d} \mu^{\prime} .
$$

In particular, if $G \equiv 1$, the uniqueness part of Lemma 2.1 shows that the solution of $(2.1,2.2)$ is $\Gamma \equiv 1$. Hence $\mathcal{R} 1=1$, and therefore the $H$ function satisfies the following nonlinear integral equation:

$$
\frac{1}{H(\mu)}=\int_{0}^{1} \frac{H\left(\mu^{\prime}\right)}{2\left(\mu+\mu^{\prime}\right)} \mu^{\prime} \mathrm{d} \mu^{\prime}
$$

This integral equation can be solved iteratively by a relaxation method in order to tabulate $H$. This presentation of Chandrasekhar's function $H$ and of the reflection operator $\mathcal{R}$ goes back to [10] (pp. 309-311, in the context of the Boltzmann equation) and is given in detail in [11] (pp. 222-224). Another presentation, based on stochastic processes, can be found in [3].

\section{The COUpling Algorithms}

In this section we give a detailed description of the coupling algorithms for the steady transport problem (1.1) with coefficients (1.3).

\subsection{Order $O(\epsilon)$ coupling}

The prescription for a coupling algorithm that approximates the solution of the interface problem to within $O(\epsilon)$ is as follows. First solve the steady transport problem

$$
\begin{aligned}
\mu \partial_{x} \Phi_{0}+\Phi_{0}-c \bar{\Phi}_{0} & =0, & (x, \mu) \in\left(x_{L}, x_{M}\right) \times[-1,1] \\
\Phi_{0}\left(x_{L}, \mu\right) & =F_{L}(\mu), & \mu \in(0,1], \\
\Phi_{0}\left(x_{M},-\mu\right) & =\mathcal{R}\left(\left.\Phi_{0}\left(x_{M}, \cdot\right)\right|_{(0,1]}\right)(\mu), & \mu \in(0,1],
\end{aligned}
$$

where $\mathcal{R}$ is the operator defined in Lemma 2.2. That the problem (3.1) has a unique solution is a more or less classical result in the theory of the transport equation: see Appendix A below. Once this is done and the density $\Phi_{0}$ has been computed, solve the diffusion problem

$$
\begin{aligned}
-\frac{1}{3} \partial_{x x} \Theta_{0}+\gamma \Theta_{0} & =0, \quad x \in\left(x_{M}, x_{R}\right), \\
\Theta_{0}\left(x_{M}\right) & =\frac{\sqrt{3}}{2} \int_{0}^{1} \mu H(\mu) \Phi_{0}\left(x_{M}, \mu\right) \mathrm{d} \mu, \\
\Theta_{0}\left(x_{R}\right) & =\frac{\sqrt{3}}{2} \int_{0}^{1} \mu H(\mu) F_{R}(\mu) \mathrm{d} \mu
\end{aligned}
$$

where $H$ is Chandrasekhar's function of defined in 2.1 .

Theorem 3.1. Let $\Psi_{\epsilon}$ be the solution of the original two-scale steady transport problem (1.1, 1.2) with coeffcients as in (1.3), and define $\Psi^{0}$ as follows:

$$
\begin{array}{ll}
\Psi^{0}(x, \mu)=\Phi_{0}(x, \mu) & \text { for }(x, \mu) \in\left(x_{L}, x_{M}\right) \times[-1,1] \\
\Psi^{0}(x, \mu)=\Theta_{0}(x) & \text { for }(x, \mu) \in\left(x_{M}, x_{R}\right) \times[-1,1] .
\end{array}
$$

Then

$$
\left\|\Psi_{\epsilon}-\Psi^{0}\right\|_{L^{1}\left(\left[x_{L}, x_{R}\right] \times[-1,1]\right)}=O(\epsilon)
$$


and, for each $x_{M}^{\prime}, x_{R}^{\prime} \in\left(x_{M}, x_{R}\right)$ such that $x_{M}^{\prime}<x_{R}^{\prime}$, one has

$$
\left\|\Psi_{\epsilon}-\Psi^{0}\right\|_{L^{2}\left(\left[x_{L}, x_{M}\right] \times[-1,1]\right)}+\left\|\Psi_{\epsilon}-\Psi^{0}\right\|_{L^{\infty}\left(\left[x_{M}^{\prime}, x_{R}^{\prime}\right] \times[-1,1]\right)}=O(\epsilon)
$$

as $\epsilon \rightarrow 0$.

Notice that the function $\Psi^{0}$ is in general discontinuous in $x$ at $x_{M}$ while $\Psi_{\epsilon}$ is continuous in $x$ for a.e. $\mu \in[-1,1]$ and each $\epsilon>0$. Hence the approximation above cannot hold in the $L^{\infty}$ norm. This discontinuity is the manifestation at the macroscopic scale of the boundary layer with thickness of order $O(\epsilon)$ at the interface inside the diffusive region. The great advantage of this procedure is that the transport region and the diffusive region are completely decoupled at this order of approximation. That may seem somewhat paradoxical: indeed, suppose that $F_{L} \equiv 0$. According to the prescription above, $\Psi^{0} \equiv 0$ in the transport region, independently of whether $F_{R} \equiv 0$ or not. However, the total flux of particles inside the diffusion region is approximated by $-\epsilon \frac{1}{3} \partial_{x} \Theta_{0}$ and gives in general a non trivial contribution of order $O(\epsilon)$ at the interface from the diffusion side. Based on this simple observation, we anticipate that the treatments of the transport and diffusion regions must be coupled somehow if one wants to get the next order of accuracy $O\left(\epsilon^{2}\right)$.

\subsection{Order $\boldsymbol{O}\left(\epsilon^{2}\right)$ coupling}

Once the solution of the problem (1.1)-(1.3) has been computed to within $O(\epsilon)$ by the prescription above, one determines the order $O(\epsilon)$ correction to the steady transport problem (3.1) by solving

$$
\begin{array}{rlrl}
\mu \partial_{x} \Phi_{1}+\Phi_{1}-c \bar{\Phi}_{1} & =0, & & (x, \mu) \in\left(x_{L}, x_{M}\right) \times[-1,1], \\
\Phi_{1}\left(x_{L}, \mu\right) & =0, & \mu \in(0,1], \\
\Phi_{1}\left(x_{M},-\mu\right) & =\mathcal{R}\left(\left.\Phi_{1}\left(x_{M}, \cdot\right)\right|_{(0,1]}\right)(\mu)+(\mu-\mathcal{R} \mu) \partial_{x} \Theta_{0}\left(x_{M}\right), & \mu \in(0,1],
\end{array}
$$

where $\lambda$ is a positive constant given by

$$
\lambda=\frac{\sqrt{3}}{2} \int \mu^{2} H(\mu) \mathrm{d} \mu .
$$

The parameter $\lambda$ is related to the so-called "extrapolated endpoints" $x_{M}-\epsilon \lambda$ and $x_{R}+\epsilon \lambda$.

Having determined $\Phi_{1}$ we next compute the diffusion approximation of the solution $\Psi_{\epsilon}$ as follows:

$$
\begin{aligned}
-\frac{1}{3} \partial_{x x} \Theta_{\epsilon}+\gamma \Theta_{\epsilon} & =0, \quad x \in\left(x_{M}, x_{R}\right), \\
\Theta_{\epsilon}\left(x_{M}\right)-\epsilon \lambda \partial_{x} \Theta_{\epsilon}\left(x_{M}\right) & =\frac{\sqrt{3}}{2} \int_{0}^{1} \mu H(\mu)\left(\Phi_{0}+\epsilon \Phi_{1}\right)\left(x_{M}, \mu\right) \mathrm{d} \mu \\
\Theta_{\epsilon}\left(x_{R}\right)+\epsilon \lambda \partial_{x} \Theta_{\epsilon}\left(x_{R}\right) & =\frac{\sqrt{3}}{2} \int_{0}^{1} \mu H(\mu) F_{R}(\mu) \mathrm{d} \mu .
\end{aligned}
$$

Once $\Phi_{0}, \Phi_{1}$ and $\Theta_{\epsilon}$ are determined, the approximation of the solution of (1.1)-(1.3) to within $O\left(\epsilon^{2}\right)$ is expressed as follows.

Theorem 3.2. Let $\Psi_{\epsilon}$ be the solution of the original two-scale steady transport problem (1.1, 1.2) with coeffcients as in (1.3). Define $\Psi_{\epsilon}^{1}$ as follows:

$$
\begin{array}{ll}
\Psi_{\epsilon}^{1}(x, \mu)=\left(\Phi_{0}+\epsilon \Phi_{1}\right)(x, \mu) & \text { for }(x, \mu) \in\left(x_{L}, x_{M}\right) \times[-1,1], \\
\Psi_{\epsilon}^{1}(x, \mu)=\Theta_{\epsilon}(x)-\epsilon \mu \partial_{x} \Theta_{\epsilon}(x) & \text { for }(x, \mu) \in\left(x_{M}, x_{R}\right) \times[-1,1] .
\end{array}
$$

Then, for each $x_{M}^{\prime}, x_{R}^{\prime} \in\left(x_{M}, x_{R}\right)$ such that $x_{M}^{\prime}<x_{R}^{\prime}$, one has

$$
\left\|\Psi_{\epsilon}-\Psi_{\epsilon}^{1}\right\|_{L^{2}\left(\left[x_{L}, x_{M}\right] \times[-1,1]\right)}+\left\|\Psi_{\epsilon}-\Psi_{\epsilon}^{1}\right\|_{L^{\infty}\left(\left[x_{M}^{\prime}, x_{R}^{\prime}\right] \times[-1,1]\right)}=O\left(\epsilon^{2}\right)
$$

as $\epsilon \rightarrow 0$. 
The following observation is crucial in understanding the coupling algorithms discussed here: a perturbation of order $O(1)$ (resp. of order $O(\epsilon)$ ) in the solution of the diffusion equation on $\left(x_{M} x_{R}\right)$ has an effect of order $O(\epsilon)$ (resp. $O\left(\epsilon^{2}\right)$ ) on the solution of the transport equation over $\left(x_{L}, x_{M}\right)$. In any case, both algorithms presented in Theorems 3.1 and 3.2 lead to approximations of the solution $\Psi_{\epsilon}$ of the original two-scale steady transport problem (1.1)-(1.3) to within $O(\epsilon)$ and $O\left(\epsilon^{2}\right)$ respectively in a finite number of steps (respectively one and two steps). By using this algorithms, one avoids iterating on the transport and diffusion solutions as is done in most other domain decomposition algorithms (e.g. in $[1,26])$.

Remark 3.1. Theorems 3.1 and 3.2 are proved below under the assumption (1.3) and (1.4) which exclude in particular the case of a purely scattering material. Our coupling algorithm still works in that case. However, this requires that the proof be modified in the following manner: the proof of Lemma 4.1, and both the estimates (4.27) and (4.32) rest on the energy estimate (4.17) in a way that requires $c$ to be less than and even bounded away from 1. If one only assumes $c=1$, the results in Lemma 4.1, (4.27) and (4.32) are still true. The argument based on the energy estimate (4.17) must be replaced by the fact that the spectrum of the unbounded operator

$$
T: f \mapsto \mu \partial_{x} f+f-\bar{f}
$$

with domain

$$
D(T)=\left\{f \in L^{2}\left(\left[x_{L}, x_{M}\right] \times[-1,1]\right) \mid \mu \partial_{x} f \in L^{2}\left(\left[x_{L}, x_{M}\right] \times[-1,1]\right), f\left(x_{L}, \mu\right)=f\left(x_{M},-\mu\right)=0, \mu \in(0,1]\right\}
$$

is the union of its continuous spectrum included in $\{z \in \mathbf{C} \mid \Re z \geq 1\}$ and of a sequence of real eigenvalues in ]0, 1[ converging to 1 (see [23]). We have chosen to present the proof based on the energy inequality (4.17) because of its wider range of applicability - in particular, $c$ does not need to be a constant as in the argument based on the explicit spectral analysis of [23]. Another argument in favor of this choice is that the $O(\epsilon)\left(\operatorname{resp} . O\left(\epsilon^{2}\right)\right)$ estimates in the statement of Theorem 3.1 (resp. 3.2) can be expressed in terms of explicitly known parameters such as $c^{*}, c_{*} \gamma_{*}, \gamma^{*}$ and $\lambda$ : see in particular the remark at the end of Section 4.

\section{ERRoR ESTIMATE FOR THE DOMAIN DECOMPOSITION ALGORITHM: THE STEADY PROBLEM}

Our proof of Theorems 3.1 and 3.2 is based on a precise error estimate for the diffusion approximation of the transport equation with boundary layer terms and on a few elementary stability results for the transport equation itself. This preliminary material is discussed in the next few sections.

\subsection{The diffusion approximation}

In the diffusive region $\left[x_{M}, x_{R}\right]$, the scaled transport equation is

$$
\mu \partial_{x} \Psi_{\epsilon}+\frac{1}{\epsilon} \Psi_{\epsilon}=\frac{1}{\epsilon}\left(1-\epsilon^{2} \gamma\right) \bar{\Psi}_{\epsilon}, \quad x_{M}<x<x_{R},-1<\mu<1 .
$$

It is supplemented with the boundary conditions

$$
\Psi_{\epsilon}\left(x_{M}, \mu\right)=F_{M}(\mu), \quad 0<\mu<1
$$

and

$$
\Psi_{\epsilon}\left(x_{R},-\mu\right)=F_{R}(\mu), \quad 0<\mu<1 .
$$

The main difficulty in the domain decomposition algorithm is that the boundary condition $F_{M}$ is not explicitly known, but eventually determined by the coupling of the transport and diffusion domains. Assuming however 
that $F_{M}$ is known, the diffusion approximation of the scaled transport equation (4.1)-(4.3) can be formulated as follows. Let $\Theta_{\epsilon}$ be the solution of the diffusion equation

$$
-\frac{1}{3} \partial_{x x} \Theta_{\epsilon}+\gamma \Theta_{\epsilon}=0, \quad x_{M}<x<x_{R}
$$

with Robin boundary conditions

$$
\begin{aligned}
\Theta_{\epsilon}-\left.\epsilon \lambda \partial_{x} \Theta_{\epsilon}\right|_{x=x_{M}} & =\frac{\sqrt{3}}{2} \int_{0}^{1} \mu H(\mu) F_{M}(\mu) \mathrm{d} \mu \\
\Theta_{\epsilon}+\left.\epsilon \lambda \partial_{x} \Theta_{\epsilon}\right|_{x=x_{R}} & =\frac{\sqrt{3}}{2} \int_{0}^{1} \mu H(\mu) F_{R}(\mu) \mathrm{d} \mu
\end{aligned}
$$

where $H$ is the Chandrasekhar function (see Def. 2.1). The Robin boundary conditions (4.5) can be viewed as Taylor expansions at order $O\left(\epsilon^{2}\right)$ of the Dirichlet boundary conditions at the extrapolated endpoints.

In particular, when $\epsilon=0$, the problem $(4.4,4.5)$ reduces to the Dirichlet problem

$$
\begin{aligned}
-\frac{1}{3} \partial_{x x} \Theta_{0}+\gamma \Theta_{0} & =0, \quad x_{M}<x<x_{R} \\
\Theta_{0}\left(x_{M}\right) & =\frac{\sqrt{3}}{2} \int_{0}^{1} \mu H(\mu) F_{M}(\mu) \mathrm{d} \mu \\
\Theta_{0}\left(x_{R}\right) & =\frac{\sqrt{3}}{2} \int_{0}^{1} \mu H(\mu) F_{R}(\mu) \mathrm{d} \mu
\end{aligned}
$$

Let $\Gamma_{\epsilon}^{M}$ be the solution of

$$
\begin{aligned}
& \mu \partial_{z} \Gamma_{\epsilon}^{M}+\Gamma_{\epsilon}^{M}-\bar{\Gamma}_{\epsilon}^{M}=0, \quad z>0,-1<\mu<1, \\
& \Gamma_{\epsilon}^{M}(0, \mu)=F_{M}(\mu)-\Theta_{\epsilon}\left(x_{M}\right)+\epsilon \mu \partial_{x} \Theta_{\epsilon}\left(x_{M}\right), \quad 0<\mu<1 \text {, }
\end{aligned}
$$

in $L^{\infty}\left([0,+\infty) ; L^{2}([-1,1],|\mu| \mathrm{d} \mu)\right.$ ) (whose existence and uniqueness is guaranteed by Lem. 2.1) while $\Gamma_{\epsilon}^{R}$ is similarly defined with the boundary data

$$
\Gamma_{\epsilon}^{R}(0, \mu)=F_{R}(\mu)-\Theta_{\epsilon}\left(x_{R}\right)-\epsilon \mu \partial_{x} \Theta_{\epsilon}\left(x_{R}\right), \quad 0<\mu<1
$$

The diffusion approximation consists then in replacing the solution $\Psi_{\epsilon}$ of the transport problem (4.1)-(4.3) by the truncated multi-scale expansion

- at order $O(\epsilon)$ :

$$
\Phi_{\epsilon}^{0}(x, \mu)=\Theta_{0}(x)+\Gamma_{0}^{M}\left(\frac{x-x_{M}}{\epsilon}, \mu\right)+\Gamma_{0}^{R}\left(\frac{x_{R}-x}{\epsilon},-\mu\right) .
$$

- at order $O\left(\epsilon^{2}\right)$ :

$$
\Phi_{\epsilon}^{1}(x, \mu)=\Theta_{\epsilon}(x)-\epsilon \mu \partial_{x} \Theta_{\epsilon}(x)+\Gamma_{\epsilon}^{M}\left(\frac{x-x_{M}}{\epsilon}, \mu\right)+\Gamma_{\epsilon}^{R}\left(\frac{x_{R}-x}{\epsilon},-\mu\right) .
$$

With these notations, the diffusion approximation for the transport equation (4.1)-(4.3) can be stated as follows.

Proposition 4.1. The error between the solution $\Psi_{\epsilon}$ of the transport problem and the truncated expansion $\Phi_{\epsilon}$ satisfies 
- At order $O(\epsilon)$ :

$$
\left\|\Psi_{\epsilon}-\Phi_{\epsilon}^{0}\right\|_{L^{\infty}\left(\left[x_{M}, x_{R}\right] \times[-1,1]\right)} \leq C_{0} \epsilon
$$

in addition

$$
\sup _{\mu \in(0,1)}\left|\Psi_{\epsilon}\left(x_{M},-\mu\right)-\mathcal{R}\left(\left.\Psi_{\epsilon}\left(x_{M}, \cdot\right)\right|_{[0,1]}\right)(\mu)\right| \leq C_{0} \epsilon .
$$

- At order $O\left(\epsilon^{2}\right)$ :

$$
\left\|\Psi_{\epsilon}-\Phi_{\epsilon}^{1}\right\|_{L^{\infty}\left(\left[x_{M}, x_{R}\right] \times[-1,1]\right)} \leq C_{1} \epsilon^{2}
$$

in addition

$$
\sup _{\mu \in(0,1)}\left|\Psi_{\epsilon}\left(x_{M},-\mu\right)-\mathcal{R}\left(\left.\Psi_{\epsilon}\left(x_{M}, \cdot\right)\right|_{[0,1]}\right)(\mu)-\epsilon(\mu-\mathcal{R} \mu) \partial_{x} \Theta_{0}\left(x_{M}\right)\right| \leq C_{1} \epsilon^{2} .
$$

In (4.12) and (4.14), $\mathcal{R}$ is the operator defined in Lemma 2.2. This Proposition is the main result in [2]; it is phrased here essentially in the notations of Theorem 2.2 in [12]. An equivalent formulation, based on stochastic processes, can be found in [3].

\subsection{Stability results}

The first stability result needed in the error estimate for the coupling algorithm bears on the transport equation itself. Let $\sigma \equiv \sigma(x)$ satisfy $0<\sigma_{*} \leq \sigma(x) \leq \sigma^{*}$ and $c \equiv c(x)$ satisfy $0<c_{*} \leq c(x) \leq c^{*}<1$ for some constants $\sigma_{*}, \sigma^{*}, c_{*}$ and $c^{*}$ and for each $x \in\left[x_{L}, x_{M}\right]$. Consider the transport equation

$$
\begin{array}{rlrl}
\mu \partial_{x} f+\sigma(f-c \bar{f}) & =0, & & (x, \mu) \in\left(x_{L}, x_{M}\right) \times[-1,1] \\
f\left(x_{L}, \mu\right) & =F_{L}(\mu), & \mu \in(0,1] ; \\
f\left(x_{M},-\mu\right) & =F_{M}(\mu), & & \mu \in(0,1] .
\end{array}
$$

We next define two operators on $L^{2}([0,1], \mu \mathrm{d} \mu)$, denoted by $\mathcal{T}_{L}$ and $\mathcal{T}_{M}$, in the following manner:

$$
\begin{gathered}
\left(\mathcal{T}_{L} F_{L}\right)(\mu)=f\left(x_{M}, \mu\right) \text { for a.e. } \mu \in(0,1] \text { when } F_{M}=0, \\
\left(\mathcal{T}_{M} F_{M}\right)(\mu)=f\left(x_{M}, \mu\right) \text { for a.e. } \mu \in(0,1] \text { when } F_{L}=0 .
\end{gathered}
$$

By linearity of the problem (4.15), in the general case where neither $F_{L}$ nor $F_{M}$ is identically 0 ,

$$
f\left(x_{M}, \mu\right)=\left(\mathcal{T}_{L} F_{L}\right)(\mu)+\left(\mathcal{T}_{M} F_{M}\right)(\mu), \quad \text { for a.e. } \mu \in(0,1]
$$

Lemma 4.1. The operators $\mathcal{T}_{L}$ and $\mathcal{T}_{M}$ are bounded on $L^{2}([0,1], \mu \mathrm{d} \mu)$; further

$$
\left\|\mathcal{T}_{L}\right\|_{\mathcal{L}\left(L^{2}([0,1], \mu \mathrm{d} \mu)\right)} \leq 1, \quad\left\|\mathcal{T}_{M}\right\|_{\mathcal{L}\left(L^{2}([0,1], \mu \mathrm{d} \mu)\right)}<1
$$

Proof. We start with the basic energy estimate obtained by multiplying (4.15) by $f$ and integrating over $\left[x_{L}, x_{R}\right] \times$ $[-1,1]$ :

$$
\begin{array}{r}
\int_{x_{L}}^{x_{M}} \int_{-1}^{1} \sigma(1-c) f^{2} \mathrm{~d} x \mathrm{~d} \mu+\int_{x_{L}}^{x_{M}} \int_{-1}^{1} \sigma c(f-\bar{f})^{2} \mathrm{~d} x \mathrm{~d} \mu+\frac{1}{2} \int_{0}^{1} \mu f\left(x_{L},-\mu\right)^{2} \mathrm{~d} \mu+\frac{1}{2} \int_{0}^{1} \mu f\left(x_{M}, \mu\right)^{2} \mathrm{~d} \mu \\
=\frac{1}{2} \int_{0}^{1} \mu F_{L}(\mu)^{2} \mathrm{~d} \mu+\frac{1}{2} \int_{0}^{1} \mu F_{M}(\mu)^{2} \mathrm{~d} \mu .
\end{array}
$$

Applying this successively to $F_{M} \equiv 0$ and $F_{L} \equiv 0$ leads to

$$
\left\|\mathcal{T}_{L}\right\|_{\mathcal{L}\left(L^{2}([0,1], \mu \mathrm{d} \mu)\right)} \leq 1 \text { and then }\left\|\mathcal{T}_{M}\right\|_{\mathcal{L}\left(L^{2}([0,1], \mu \mathrm{d} \mu)\right)} \leq 1
$$


It remains to prove that the second inequality is strict. Set $F_{L} \equiv 0$. The energy estimate (4.17) and the bounds assumed on the functions $\sigma \equiv \sigma(x)$ and $c \equiv c(x)$ imply that

$$
\nu\left(\|f-\bar{f}\|_{L^{2}\left(\left[x_{L}, x_{M}\right] \times[-1,1]\right)}^{2}+\|f\|_{L^{2}\left(\left[x_{L}, x_{M}\right] \times[-1,1]\right)}^{2}\right) \leq \frac{1}{2}\|\phi\|_{L^{2}([0,1], \mu \mathrm{d} \mu)}^{2}-\frac{1}{2}\left\|\mathcal{T}_{M} \phi\right\|_{L^{2}([0,1], \mu \mathrm{d} \mu)}^{2}
$$

where $\nu=\sigma^{*} \min \left(1-c^{*}, c_{*}\right)$. On the other hand, since $F_{L} \equiv 0$, for a.e. $\mu \in(0,1]$ one has

$$
\begin{aligned}
\frac{1}{2} \mu\left(\mathcal{T}_{M} \phi(\mu)\right)^{2} & =\int_{x_{L}}^{x_{M}} f(x, \mu) \mu \partial_{x} f(x, \mu) \mathrm{d} x \\
& \leq\left\|\mu \partial_{x} f(\cdot, \mu)\right\|_{L^{2}\left(\left[x_{L}, x_{M}\right]\right.}\|f(\cdot, \mu)\|_{L^{2}\left(\left[x_{L}, x_{M}\right]\right)} .
\end{aligned}
$$

Integrating this in $\mu$ over $(0,1]$, one finds

$$
\begin{aligned}
\frac{1}{2}\left\|\mathcal{T}_{M} \phi\right\|_{L^{2}([0,1], \mu \mathrm{d} \mu)}^{2} \leq & \left\|\mu \partial_{x} f\right\|_{L^{2}\left(\left[x_{L}, x_{M}\right] \times[-1,1]\right)}\|f\|_{L^{2}\left(\left[x_{L}, x_{M}\right] \times[-1,1]\right)} \\
\leq & \left(\|\sigma c(f-\bar{f})\|_{L^{2}\left(\left[x_{L}, x_{M}\right] \times[-1,1]\right)}\right. \\
& \left.+\|\sigma(1-c) f\|_{\left.L^{2}\left(\left[x_{L}, x_{M}\right] \times[-1,1]\right)\right)}\right)\|f\|_{L^{2}\left(\left[x_{L}, x_{M}\right] \times[-1,1]\right)} .
\end{aligned}
$$

By using (4.19) and (4.20) one arrives at

$$
\left\|\mathcal{T}_{M} \phi\right\|_{L^{2}([0,1], \mu \mathrm{d} \mu)}^{2} \leq \frac{3 \sigma^{*}}{2 \nu}\left(\|\phi\|_{L^{2}([0,1], \mu \mathrm{d} \mu)}^{2}-\|\mathcal{T} \phi\|_{L^{2}([0,1], \mu \mathrm{d} \mu)}^{2}\right)
$$

and since this is true of any function $\phi \in L^{2}([0,1], \mu \mathrm{d} \mu)$, one eventually obtains

$$
\left\|\mathcal{T}_{M}\right\|_{\mathcal{L}\left(L^{2}([0,1], \mu \mathrm{d} \mu)\right.} \leq \sqrt{\frac{3 \sigma^{*}}{2 \nu+3 \sigma^{*}}}<1
$$

which concludes the proof.

The second stability result that we need bears on the operator $\mathcal{R}$ defined in Lemma 2.2 and can be seen as a stability result for the transport equation, however inside the diffusive region.

Lemma 4.2. The operator $\mathcal{R}$ defined in Lemma 2.2 is a contraction mapping on the space $L^{2}([0,1], \mu \mathrm{d} \mu)$ :

$$
\|\mathcal{R}\|_{\mathcal{L}\left(L^{2}([0,1], \mu \mathrm{d} \mu)\right)}=1 .
$$

Proof. The energy estimate analogous to (4.17) for the problem (2.1) with the boundary condition (2.2) is obtained by multiplying equation (2.1) by $\Gamma$, on account of (2.2) and of the asymptotic behavior as $z \rightarrow+\infty$ in Lemma 2.1. One finds that

$$
\int_{0}^{+\infty} \int_{-1}^{1}(\Gamma-\bar{\Gamma})^{2} \mathrm{~d} z \mathrm{~d} \mu+\frac{1}{2} \int_{0}^{1} \mu \Gamma\left(x_{L},-\mu\right)^{2} \mathrm{~d} \mu=\frac{1}{2} \int_{0}^{1} \mu G(\mu)^{2} \mathrm{~d} \mu .
$$

Hence

$$
\frac{1}{2} \int_{0}^{1} \mu \Gamma\left(x_{L},-\mu\right)^{2} \mathrm{~d} \mu \leq \frac{1}{2} \int_{0}^{1} \mu G(\mu)^{2} \mathrm{~d} \mu
$$

which means that $\|\mathcal{R}\|_{\mathcal{L}\left(L^{2}([0,1], \mu \mathrm{d} \mu)\right)} \leq 1$. This inequality is actually an equality since $\mathcal{R} 1=1$. 


\subsection{Continuity across the interface}

The last ingredient in our proof is the following continuity statement.

Lemma 4.3. Let $\sigma \equiv \sigma(x)$ satisfy $0<\sigma_{*} \leq \sigma(x) \leq \sigma^{*}$ and $c \equiv c(x)$ satisfy $0 \leq c(x) \leq c_{*}<1$ for some constants $\sigma_{*}, \sigma^{*}$ and $c^{*}$ and for each $x \in\left[x_{L}, x_{R}\right]$. Consider the transport equation

$$
\begin{aligned}
2 \mu \partial_{x} f+\sigma(f-c \bar{f}) & =0, & (x, \mu) \in\left(x_{L}, x_{R}\right) \times[-1,1] ; \\
f\left(x_{L}, \mu\right) & =F_{L}(\mu), & \mu \in(0,1] ; \\
f\left(x_{R},-\mu\right) & =F_{R}(\mu), & \mu \in(0,1],
\end{aligned}
$$

where $F_{L}$ and $F_{M} \in L^{2}([0,1], \mu \mathrm{d} \mu)$. Then, for a.e. $\mu \in[-1,1]$, the function $x \mapsto f(x, \mu)$ is continuous.

Proof. The energy estimate (4.17) implies the existence of a positive constant $C$ such that

$$
\int_{x_{L}}^{x_{M}} \int_{-1}^{1} \sigma(1-c) f^{2} \mathrm{~d} x \mathrm{~d} \mu \leq C \text { and } \int_{x_{L}}^{x_{M}} \int_{-1}^{1} \sigma c(f-\bar{f})^{2} \mathrm{~d} x \mathrm{~d} \mu \leq C
$$

These inequalities and the transport equation (4.23) imply that

$$
\mu \partial_{x} f=-\sigma c(f-\bar{f})-\sigma(1-c) f \in L^{2}\left(\left[x_{L}, x_{R}\right] \times[-1,1]\right)
$$

because of the bounds $0 \leq \sigma \leq \sigma^{*}$ and $0 \leq c \leq 1$. This implies in particular that the derivative $\partial_{x} f(\cdot, \mu) \in$ $L^{2}\left(\left[x_{L}, x_{R}\right]\right)$ for a.e. $\mu \in[-1,1]$, which in turn establishes the announced continuity.

\subsection{Proof of Theorem 3.1}

Apply Lemma 4.3 with

$$
\sigma(x)=\mathbf{1}_{\left[x_{L}, x_{M}\right](x)}+\frac{1}{\epsilon} \mathbf{1}_{\left[x_{M}, x_{R}\right]}(x) \text { and } c(x)=1-\epsilon^{2} \gamma \text { for } x \in\left[x_{M}, x_{R}\right] .
$$

Hence, for each $\epsilon \in\left(0, \epsilon^{*}\right)$ and a.e. $\mu \in[-1,1]$, the function $x \mapsto \Psi_{\epsilon}(x, \mu)$ is continuous — see (1.4) and (1.5) for the definition of $\epsilon^{*}$. Notice that this continuity is in general not uniform in $\epsilon$ for $\sigma$ is not bounded uniformly in $\epsilon$ in the diffusive region. This lack of uniformity is consistent with the presence of boundary layers $\Gamma_{0}^{M}$ and $\Gamma_{0}^{R}$ with variations of order 1 over lengths of order $\epsilon$ in the truncated expansions (4.9). For the $O(\epsilon)$ estimate, this continuity at $x_{M}$ for a.e. $\mu \in[-1,0)$ together with (4.12) implies that

$$
\phi_{\epsilon}(\mu):=\Psi_{\epsilon}\left(x_{M},-\mu\right)-\mathcal{R}\left(\left.\Psi_{\epsilon}\left(x_{M}, \cdot\right)\right|_{(0,1]}\right)(\mu) \text { satisfies }\left\|\phi_{\epsilon}\right\|_{L^{\infty}(0,1)} \leq C_{0} \epsilon .
$$

Then, by definition of the operators $\mathcal{T}_{L}, \mathcal{T}_{M}$ and $\mathcal{R}$, one has

$$
\Psi_{\epsilon}\left(x_{M}, \mu\right)=\left(\mathcal{T}_{L} F_{L}\right)(\mu)+\left(\left.\mathcal{T}_{M} \Psi_{\epsilon}\left(x_{M}, \cdot\right)\right|_{[-1,0)}\right)(\mu), \quad \mu \in(0,1]
$$

and hence

$$
\left.\Psi_{\epsilon}\left(x_{M}, \cdot\right)\right|_{[-1,0)}=\mathcal{R} \circ \mathcal{T}_{L} F_{L}+\left.\mathcal{R} \circ \mathcal{T}_{M} \Psi_{\epsilon}\left(x_{M}, \cdot\right)\right|_{[-1,0)}+\phi_{\epsilon}
$$

By Lemmas 4.1 and $4.2\left\|\mathcal{R} \circ \mathcal{T}_{M}\right\|_{\mathcal{L}\left(L^{2}([0,1], \mu \mathrm{d} \mu)\right.}<1$; hence $I-\mathcal{R} \circ \mathcal{T}_{M}$ is invertible in $\mathcal{L}\left(L^{2}([0,1], \mu \mathrm{d} \mu)\right)$ and

$$
\left.\Psi_{\epsilon}\left(x_{M}, \cdot\right)\right|_{[-1,0)}=\left(I-\mathcal{R} \circ \mathcal{T}_{M}\right)^{-1}\left(\mathcal{R} \circ \mathcal{T}_{L} F_{L}+\phi_{\epsilon}\right)
$$

Define $\chi_{0}$ by

$$
\chi_{0}=\left(I-\mathcal{R} \circ \mathcal{T}_{M}\right)^{-1} \mathcal{R} \circ \mathcal{T}_{L} F_{L}
$$


Because of (4.24) and the stability results in Lemmas 4.1 and 4.2

$$
\left\|\left.\Psi_{\epsilon}\left(x_{M}, \cdot\right)\right|_{[-1,0)}-\chi_{0}\right\|_{L^{2}(\mu \mathrm{d} \mu)} \leq \frac{\left\|\phi_{\epsilon}\right\|_{L^{\infty}}}{1-\left\|\mathcal{T}_{M}\right\|_{\mathcal{L}\left(L^{2}(\mu \mathrm{d} \mu)\right)}} \leq \frac{C_{0} \epsilon}{1-\left\|\mathcal{T}_{M}\right\|_{\mathcal{L}\left(L^{2}(\mu \mathrm{d} \mu)\right)}} .
$$

Consider $\Phi$ the solution of

$$
\begin{array}{rlrl}
\mu \partial_{x} \Phi+(\Phi-c \bar{\Phi}) & =0, & & (x, \mu) \in\left(x_{L}, x_{M}\right) \times[-1,1] ; \\
\Phi\left(x_{L}, \mu\right) & =F_{L}(\mu), & \mu \in(0,1] ; \\
\Phi\left(x_{M},-\mu\right) & =\chi_{0}(\mu), & \mu \in(0,1] .
\end{array}
$$

Applying the energy estimate (4.17) shows that

$$
\left\|\Psi_{\epsilon}-\Phi\right\|_{L^{2}\left(\left[x_{L}, x_{M}\right] \times[-1,1]\right)}=O(\epsilon) .
$$

On the other hand, the definition of $\chi_{0}$ implies that $\Phi=\Phi_{0}$ (the solution of (3.1)). This establishes the $O(\epsilon)$ approximation for the transport domain.

Let $N_{\epsilon}$ be the solution of

$$
\begin{aligned}
-\frac{1}{3} \partial_{x x} N_{\epsilon}+\gamma N_{\epsilon} & =0, \quad x \in\left(x_{M}, x_{R}\right) ; \\
N_{\epsilon}\left(x_{M}\right) & =\frac{\sqrt{3}}{2} \int_{0}^{1} \mu H(\mu) \Psi_{\epsilon}\left(x_{M}, \mu\right) \mathrm{d} \mu ; \\
N_{\epsilon}\left(x_{R}\right) & =\frac{\sqrt{3}}{2} \int_{0}^{1} \mu H(\mu) F_{R}(\mu) \mathrm{d} \mu .
\end{aligned}
$$

By (4.18) and (4.26), $\left|N_{\epsilon}\left(x_{M}\right)-\Theta_{0}\left(x_{M}\right)\right|=O(\epsilon)$ and hence, by the Maximum Principle for the diffusion equation $\left\|N_{\epsilon}-\Theta_{0}\right\|_{L^{\infty}\left(\left[x_{M}, x_{R}\right]\right)}=O(\epsilon)$. On the other hand, by the order $O(\epsilon)$ statement in Proposition 4.1, one finds that $\left\|\Psi_{\epsilon}-N_{\epsilon}\right\|_{L^{1}\left(\left[x_{M}, x_{R}\right] \times[-1,1]\right)}=O(\epsilon)$ and that, for any choice of interior points $x_{M}^{\prime}<x_{R}^{\prime}$ in $\left(x_{M}, x_{R}\right)$, $\left\|\Psi_{\epsilon}-N_{\epsilon}\right\|_{L^{\infty}\left(\left[x_{M}^{\prime}, x_{R}^{\prime}\right] \times[-1,1]\right)}=O(\epsilon)$. This eventually implies that

$$
\left\|\Psi_{\epsilon}-\Theta_{0}\right\|_{L^{1}\left(\left[x_{M}, x_{R}\right] \times[-1,1]\right)}=O(\epsilon)
$$

and

$$
\left\|\Psi_{\epsilon}-\Theta_{0}\right\|_{L^{\infty}\left(\left[x_{M}^{\prime}, x_{R}^{\prime}\right] \times[-1,1]\right)}=O(\epsilon),
$$

which is precisely the $O(\epsilon)$ approximation for the diffusive part of the domain.

\subsection{Proof of Theorem 3.2}

The proof of the $O\left(\epsilon^{2}\right)$ approximation is essentially identical to the $O(\epsilon)$ case. For a.e. $\mu \in[-1,0), x \mapsto$ $\Psi_{\epsilon}(x, \mu)$ is continuous at $x_{M}$ and hence, by (4.14), the function $\psi_{\epsilon}$ defined by

$$
\psi_{\epsilon}(\mu):=\Psi_{\epsilon}\left(x_{M},-\mu\right)-\mathcal{R}\left(\left.\Psi_{\epsilon}\left(x_{M}, \cdot\right)\right|_{(0,1]}\right)(\mu)-\epsilon(\mu-\mathcal{R} \mu) \partial_{x} \Theta_{0}\left(x_{M}\right)
$$

satisfies

$$
\left\|\psi_{\epsilon}\right\|_{L^{\infty}([0,1])} \leq C_{1} \epsilon^{2}
$$

Hence

$$
\left.\Psi_{\epsilon}\left(x_{M}, \cdot\right)\right|_{[-1,0)}=\mathcal{R} \circ \mathcal{T}_{L} F_{L}+\left.\mathcal{R} \circ \mathcal{T}_{M} \Psi_{\epsilon}\left(x_{M}, \cdot\right)\right|_{[-1,0)}+\epsilon(\mu-\mathcal{R} \mu) \partial_{x} \Theta_{0}\left(x_{M}\right)+\psi_{\epsilon},
$$


which we put in the form

$$
\left.\Psi_{\epsilon}\left(x_{M}, \cdot\right)\right|_{[-1,0)}=\left(I-\mathcal{R} \circ \mathcal{T}_{M}\right)^{-1}\left(\mathcal{R} \circ \mathcal{T}_{L} F_{L}+\epsilon(\mu-\mathcal{R} \mu) \partial_{x} \Theta_{0}\left(x_{M}\right)+\psi_{\epsilon}\right)
$$

Define $\chi_{\epsilon}$ by

$$
\chi_{\epsilon}=\left(I-\mathcal{R} \circ \mathcal{T}_{M}\right)^{-1}\left(\mathcal{R} \circ \mathcal{T}_{L} F_{L}+\epsilon(\mu-\mathcal{R} \mu) \partial_{x} \Theta_{0}\left(x_{M}\right)\right) .
$$

Because of (4.28) and the stability results in Lemmas 4.1 and 4.2

$$
\left\|\left.\Psi_{\epsilon}\left(x_{M}, \cdot\right)\right|_{[-1,0)}-\chi_{\epsilon}\right\|_{L^{2}(\mu \mathrm{d} \mu)} \leq \frac{\left\|\psi_{\epsilon}\right\|_{L^{\infty}}}{1-\left\|\mathcal{T}_{M}\right\|_{\mathcal{L}\left(L^{2}(\mu \mathrm{d} \mu)\right)}} \leq \frac{C_{1} \epsilon^{2}}{1-\left\|\mathcal{T}_{M}\right\|_{\mathcal{L}\left(L^{2}(\mu \mathrm{d} \mu)\right)}}
$$

Let $\Phi_{\epsilon}$ be the solution of

$$
\begin{aligned}
\mu \partial_{x} \Phi_{\epsilon}+\Phi_{\epsilon}-c \overline{\Phi_{\epsilon}} & =0, \quad(x, \mu) \in\left(x_{L}, x_{M}\right) \times[-1,1] \\
\Phi_{\epsilon}\left(x_{L}, \mu\right) & =F_{L}(\mu), \quad \mu \in(0,1] \\
\Phi_{\epsilon}\left(x_{M},-\mu\right) & =\chi_{\epsilon}(\mu), \quad \mu \in(0,1]
\end{aligned}
$$

Applying the energy estimate (4.17) shows that

$$
\left\|\Psi_{\epsilon}-\Phi_{\epsilon}\right\|_{L^{2}\left(\left[x_{L}, x_{M}\right] \times[-1,1]\right)}=O\left(\epsilon^{2}\right) .
$$

On the other hand, the definition of $\chi_{\epsilon}$ implies that the boundary condition at $x_{M}$ for $\Phi_{\epsilon}$ can be replaced by

$$
\Phi_{\epsilon}\left(x_{M},-\mu\right)=\mathcal{R}\left(\left.\Phi_{\epsilon}\left(x_{M}, \cdot\right)\right|_{(0,1]}\right)(\mu)+\epsilon(\mu-\mathcal{R} \mu) \partial_{x} \Theta_{0}\left(x_{M}\right), \quad \mu \in(0,1]
$$

By linearity of the problem (4.31) and uniqueness of the solution to this problem with the above boundary condition at $x_{M}$, one finds that $\Phi_{\epsilon}=\Phi_{0}+\epsilon \Phi_{1}$ which proves the $O\left(\epsilon^{2}\right)$ approximation for the transport domain.

Let $\tilde{N}_{\epsilon}$ be the solution of

$$
\begin{aligned}
-\frac{1}{3} \partial_{x x} \tilde{N}_{\epsilon}+\gamma \tilde{N}_{\epsilon} & =0, \quad x \in\left(x_{M}, x_{R}\right) \\
\tilde{N}_{\epsilon}\left(x_{M}\right)-\epsilon \lambda \partial_{x} \tilde{N}_{\epsilon}\left(x_{M}\right) & =\frac{\sqrt{3}}{2} \int_{0}^{1} \mu^{2} H(\mu) \Psi_{\epsilon}\left(x_{M}, \mu\right) \mathrm{d} \mu \\
\tilde{N}_{\epsilon}\left(x_{R}\right)+\epsilon \lambda \partial_{x} \tilde{N}_{\epsilon}\left(x_{R}\right) & =\frac{\sqrt{3}}{2} \int_{0}^{1} \mu^{2} H(\mu) F_{R}(\mu) \mathrm{d} \mu .
\end{aligned}
$$

By (4.18), (4.30) and the $L^{\infty}$ stability of the diffusion equation with Robin boundary conditions, $\left\|\tilde{N}_{\epsilon}-\Theta_{\epsilon}\right\|_{L^{\infty}\left(\left[x_{M}, x_{R}\right]\right)}=O\left(\epsilon^{2}\right)$. By the order $O\left(\epsilon^{2}\right)$ statement in Proposition 4.1, for any choice of interior points $x_{M}^{\prime}<x_{R}^{\prime}$ in $\left(x_{M}, x_{R}\right),\left\|\Psi_{\epsilon}-\tilde{N}_{\epsilon}\right\|_{L^{\infty}\left(\left[x_{M}^{\prime}, x_{R}^{\prime}\right] \times[-1,1]\right)}=O\left(\epsilon^{2}\right)$. This eventually implies that

$$
\left\|\Psi_{\epsilon}-\Theta_{\epsilon}\right\|_{L^{\infty}\left(\left[x_{M}^{\prime}, x_{R}^{\prime}\right] \times[-1,1]\right)}=O\left(\epsilon^{2}\right),
$$

which is precisely the $O\left(\epsilon^{2}\right)$ approximation for the diffusion part of the domain.

Remark 4.1. By using the basic energy estimate (4.17), the bound (4.21) and the techniques in [12] — see in particular estimates (2.10) and (2.13) there - one can replace the $O(\epsilon)$ in Theorem 3.1 or $O\left(\epsilon^{2}\right)$ in Theorem 3.1 by terms of the form $C_{0} \epsilon$ and $C_{1} \epsilon$ respectively, where $C_{0}$ and $C_{1}$ have explicit expressions in terms of data such as $c^{*}, c_{*}, \gamma^{*}, \gamma_{*}, \lambda, x_{M}-x_{L}, x_{R}-x_{M}$ and various norms of $F_{L}$ and $F_{R}$. 


\section{Extensions}

\subsection{The time-dependent problem}

In many applications of transport theory, one has to deal with evolution rather than steady problems. Hence there is some interest in extending the domain decomposition algorithms studied above to time-dependent problems. There is however a fundamental difficulty in doing so: the natural time-scale of the diffusion equation - in the domain $\left(x_{M}, x_{R}\right)$ - is much longer than that of the transport equation - in the domain $\left(x_{L}, x_{M}\right)$. One way of avoiding this difficulty is to prepare the initial and boundary data so that the evolution time scale in the transport region is exactly as long as in the diffusive region. In particular, this requires slowly varying boundary data, and an initial data that almost belongs to the null-space of the transport operator. Keeping the same coefficients as in (1.3), we start from the problem

$$
\begin{aligned}
& \epsilon \partial_{t} \Psi_{\epsilon}+\mu \partial_{x} \Psi_{\epsilon}+\sigma \Psi_{\epsilon}=\sigma c \bar{\Psi}_{\epsilon}, \quad t>0, \quad(x, \mu) \in\left(x_{L}, x_{M}\right) \times[-1,1], \\
& \Psi_{\epsilon}\left(t, x_{L}, \mu\right)=F_{L}(t, \mu), \quad t>0, \quad \mu \in(0,1], \\
& \Psi_{\epsilon}\left(t, x_{R},-\mu\right)=F_{R}(t, \mu), \quad t>0, \quad \mu \in(0,1], \\
& \Psi_{\epsilon}(0, x, \mu)=\Psi_{I}(x, \mu), \quad(x, \mu) \in\left(x_{L}, x_{M}\right) \times[-1,1],
\end{aligned}
$$

assuming the compatibility conditions

$$
\begin{aligned}
& \mu \partial_{x} \Psi_{I}+\sigma(x) \Psi_{I}=\sigma(x) c(x) \bar{\Psi}_{I}, \quad x_{L}<x<x_{M}, \quad \mu \in[-1,1], \\
& \Psi_{I} \equiv \Psi_{I}(x), \quad x_{M}<x<x_{R}, \quad \mu \in[-1,1], \\
& F_{L}(0, \mu)=\Psi_{I}\left(x_{L}, \mu\right), \quad \mu \in(0,1], \\
& F_{R}(0, \mu)=\Psi_{I}\left(x_{R}\right), \quad \mu \in(0,1] \text {. }
\end{aligned}
$$

The time-dependent coupling algorithm at order $O(\epsilon)$ is as follows:

- on the transport region $x_{L}<x<x_{M}$, solve

$$
\begin{aligned}
\epsilon \partial_{t} \Phi_{\epsilon}+\mu \partial_{x} \Phi_{\epsilon}+\sigma \Phi_{\epsilon} & =\sigma c \bar{\Phi}_{\epsilon} & & \\
\Phi_{\epsilon}\left(t, x_{L}, \mu\right) & =F_{L}(t, \mu), & & 0<\mu<1, \\
\Phi_{\epsilon}\left(t, x_{M},-\mu\right) & =\mathcal{R}\left(\left.\Phi_{\epsilon}\left(t, x_{M}, \cdot\right)\right|_{(0,1]}\right)(\mu), & & 0<\mu<1, \\
\Phi_{\epsilon}(0, x, \mu) & =\Psi_{I}(x, \mu), & &
\end{aligned}
$$

where $\mathcal{R}$ is the operator defined in Lemma 2.2;

- on the diffusion region $x_{M}<x<x_{R}$, solve

$$
\begin{aligned}
\partial_{t} \Theta_{\epsilon} & =\frac{1}{3} \partial_{x x} \Theta_{\epsilon}-\gamma \Theta_{\epsilon}, \\
\left.\Theta_{\epsilon}\right|_{x=x_{M}} & =\frac{\sqrt{3}}{2} \int_{0}^{1} \mu H(\mu) \Phi_{\epsilon}\left(t, x_{M}, \mu\right) \mathrm{d} \mu, \\
\left.\Theta_{\epsilon}\right|_{x=x_{R}} & =\frac{\sqrt{3}}{2} \int_{0}^{1} \mu H(\mu) F_{R}(t, \mu) \mathrm{d} \mu, \\
\left.\Theta_{\epsilon}\right|_{t=0} & =\Psi_{I},
\end{aligned}
$$

where $H$ is Chandrasekhar's function — see Definition 2.1; 
- eventually the global solution is approximated by

$$
\begin{array}{ll}
\Psi_{\epsilon}(t, x, \mu)=\Phi_{\epsilon}(t, x, \mu), & x_{L}<x<x_{M}, \\
\Psi_{\epsilon}(t, x, \mu)=\Theta_{\epsilon}(t, x), & x_{M}<x<x_{R} .
\end{array}
$$

A few remarks on this domain decomposition method are in order. First, because of the assumption bearing on the boundary and initial data, if

$$
\partial_{t} F_{L} \in L^{2}([0, T] \times[0,1], \mu \mathrm{d} t \mathrm{~d} \mu) \text { for all } T>0
$$

the energy estimate implies that

$$
\partial_{t} \Phi_{\epsilon}=O(1) \text { in } L^{2}\left([0, T] \times\left[x_{L}, x_{M}\right] \times[-1,1], \mathrm{d} t \mathrm{~d} x \mathrm{~d} \mu\right) \text { for all } T>0
$$

Using (5.3) implies that the boundary data for the diffusion equation is uniformly bounded in $H_{l o c}^{1}([0,+\infty))$ since

$$
\left.\partial_{t} \Phi_{\epsilon}\right|_{x=x_{M}}=O(1) \text { in } L^{2}([0, T] \times[-1,1], \mu \mathrm{d} t \mathrm{~d} \mu) .
$$

In particular, the (slow) time scale of the diffusion equation is consistent with the (slow) time scale of the distribution of particles emerging from the transport region. A straightforward modification of (4.9) leads to a boundary layer governed by $(2.1,2.2)$ - exactly as in the steady case. Secondly, the transport and diffusive regions are decoupled globally in time up to an $O(\epsilon)$ error by using the appropriate reflection condition at the interface, that is given in terms of the operator $\mathcal{R}$. One can see that in the other existing domain decomposition algorithms, there is an iteration within any given time step so as to match the transport and diffusive domains. As already mentioned above, avoiding these iterations is one of the benefits of using the exact asymptotic reflection operator $\mathcal{R}$ instead of other, ad hoc prescriptions for the transmission condition at the interface.

\subsection{The case of higher space dimensions}

The methods in this paper can also be extended to transport equations in space dimensions higher than 1. Consider for example the transport equation with isotropic scattering

$$
\begin{aligned}
\omega \cdot \nabla_{x} \Psi(x, \omega)+\sigma(x) \Psi(x, \omega) & =\sigma(x) c(x) \bar{\Psi}(x), \\
\text { where } \bar{\Psi}(x) & =\frac{1}{\left|\mathbf{S}^{D-1}\right|} \int_{\mathbf{S}^{D-1}} \Psi(x, \omega) \mathrm{d} \omega,
\end{aligned}
$$

posed for $(x, \omega) \in \Omega \times \mathbf{S}^{D-1}$, where $\Omega$ is a smooth, bounded convex domain in $\mathbf{R}^{D}$. The coefficients $\sigma$ are of the form

$$
\begin{aligned}
& \sigma(x)=\sigma_{T}(x), \quad \text { and } \quad c(x)=c_{T}(x) \in(0,1), \quad \text { for } x \in \Omega_{T} \text {, } \\
& \sigma(x)=\epsilon^{-1} \sigma_{D}(x), \quad \text { and } \quad c(x)=1-\epsilon^{2} \gamma(x), \quad \text { for } x \in \Omega_{D} .
\end{aligned}
$$

Here $\Omega_{D}$ is a smooth, convex domain whose closure $\bar{\Omega}_{D}$ is included in $\Omega$, and $\Omega_{T}=\Omega \backslash \bar{\Omega}_{D}$. The functions $\sigma_{T}$, $\sigma_{D}, \gamma$ are assumed to be smooth and positive; likewise, the function $c_{T}$ is assumed to be smooth.

The problem (5.6) is supplemented with the boundary condition

$$
\Psi(x, \omega)=F_{b}(x, \omega), \quad \text { for }(x, \omega) \in \Sigma^{-} .
$$

Here

$$
\Sigma^{-}=\left\{(x, \omega) \in \partial \Omega \times \mathbf{S}^{D-1} \mid \omega \cdot n_{x}<0\right\},
$$




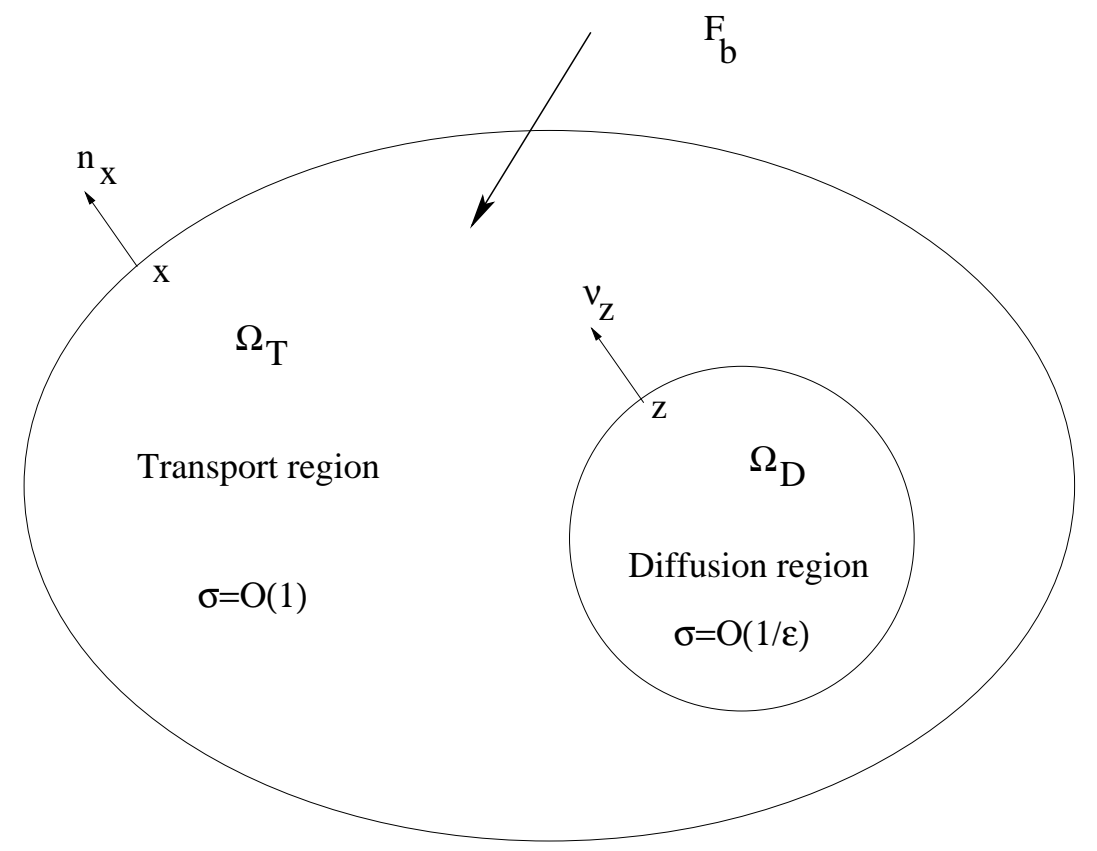

FIGURE 2. Geometry of the multi-dimensional interface problem.

where $n_{x}$ is the unit outward normal at the point $x$ of $\partial \Omega$. In this problem, the interface is $\partial \Omega_{D}$ (see Fig. 2); further, we need the notations

$$
\begin{aligned}
& \Sigma_{i}^{+}=\left\{(x, \omega) \in \partial \Omega_{D} \times \mathbf{S}^{D-1} \mid \omega \cdot \nu_{x}>0\right\}, \\
& \Sigma_{i}^{-}=\left\{(x, \omega) \in \partial \Omega_{D} \times \mathbf{S}^{D-1} \mid \omega \cdot \nu_{x}<0\right\},
\end{aligned}
$$

where $\nu_{x}$ is the unit outward normal to $\Omega_{D}$ at the point $x \in \partial \Omega_{D}$. We first describe the analog of Chandrasekhar's $\mathrm{H}$-function and of the boundary layer response operator $\mathcal{R}$ for this problem. Given $\nu \in \mathbf{S}^{D-1}$, consider the auxiliary problem

$$
\begin{aligned}
-(\omega \cdot \nu) \partial_{z} \Gamma+\Gamma-\bar{\Gamma} & =0, & & z>0, \omega \in \mathbf{S}^{D-1} \\
\Gamma(0, \omega) & =G(\omega), & & \omega \cdot \nu<0 .
\end{aligned}
$$

Denote by $\mathbf{S}_{\nu}^{-}=\left\{\omega \in \mathbf{S}^{D-1} \mid \omega \cdot \nu<0\right\}$. Proceeding exactly as in Section 2, one sees that, given $G \in$ $L^{2}\left(\mathbf{S}_{\nu}^{-},|\omega \cdot \nu| \mathrm{d} \omega\right)$, the problem (5.11) has a unique solution $\Gamma \in L^{\infty}\left(\mathbf{R}_{+} ; L^{2}\left(\mathbf{S}^{D-1},|\omega \cdot \nu| \mathrm{d} \omega\right)\right)$. In addition, there exists a linear functional $\Lambda_{\nu}$ and a bounded operator $\mathcal{R}_{\nu}$ both acting on $L^{2}\left(\mathbf{S}_{\nu}^{-},|\omega \cdot \nu| \mathrm{d} \omega\right)$ such that

$$
\begin{aligned}
\Gamma(z, \omega) \rightarrow \Gamma_{\infty} & =\Lambda_{\nu}(G) & & \text { for each } \omega \in \mathbf{S}^{D-1} \text { and } \\
\Gamma\left(0, \mathbf{s}_{\nu}(\omega)\right) & =\left(\mathcal{R}_{\nu} G\right)(\omega) & & \text { for each } \omega \in \mathbf{S}_{\nu}^{-},
\end{aligned}
$$

with the notation $\mathbf{s}_{\nu}(\omega)=\omega-2(\omega \cdot \nu) \nu$. The linear functional $\Lambda_{\nu}$ and the operator $\mathcal{R}_{\nu}$ are expressed in terms of a variant of the Chandrasekhar $H$-function which can be computed numerically by solving a nonlinear integral 
equation that is analogous to (2.6): see Appendix B. The coupling algorithm at order $O(\epsilon)$ for this problem is as follows:

- on the transport domain $\Omega_{T}$, solve

$$
\begin{aligned}
\omega \cdot \nabla_{x} \Phi(x, \omega)+\sigma_{T}(x) \Phi(x, \omega) & =\sigma_{T}(x) c_{T}(x) \bar{\Phi}(x), & & (x, \omega) \in \Omega_{T} \times \mathbf{S}^{D-1} \\
\Phi(x, \omega) & =F_{b}(x, \omega), & & (x, \omega) \in \Sigma^{-} \\
\Phi\left(x, \mathbf{s}_{\nu_{x}}(\omega)\right) & =\mathcal{R}_{\nu_{x}} \Phi(x, \omega), & & (x, \omega) \in \Sigma_{i}^{+}
\end{aligned}
$$

- on the diffusion domain $\Omega_{D}$, solve

$$
\begin{aligned}
\gamma(x) \Theta(x)-\frac{1}{D} \nabla_{x} \cdot\left(\frac{1}{\sigma_{D}(x)} \nabla_{x} \Theta(x)\right) & =0, & & \text { on } \Omega_{D}, \\
\Theta(x) & =\Lambda_{\nu_{x}}(\Phi(x, \cdot)), & & \text { on } \partial \Omega_{T}
\end{aligned}
$$

- finally the solution $\Psi_{\epsilon}$ of (5.6)-(5.8) on $\Omega \times \mathbf{S}^{D-1}$ is approximated to within $O(\epsilon)$ by

$$
\begin{array}{ll}
\Psi_{\epsilon}(x, \omega)=\Phi(x, \omega) & \text { if } x \in \Omega_{T}, \\
\Psi_{\epsilon}(x, \omega)=\Theta(x) & \text { if } x \in \Omega_{D} .
\end{array}
$$

There is also a $O\left(\epsilon^{2}\right)$ coupling, whose formulation follows essentially Theorem 3.2, and which can be easily worked out with the material presented in this paper.

\subsection{The discrete-ordinate method}

We return to the steady problem $(1.1,1.2)$ with coefficients as in $(1.3)$, and discuss the domain decomposition algorithms for the discrete-ordinate method. The discrete-ordinate method is a semi-discrete version of (1.1) where only the angle variable $\mu$ is discretized. In this method, the variable $\mu$ is discretized by a set $\mathcal{M}$ of $2 M$ quadrature points $\mu_{m}$ with quadrature weights $\alpha_{m}>0$. These points and weights are indexed by $m \in \mathcal{M}=\{-M, \ldots,-1,1, \ldots, M\}$ ordered as follows:

$$
-1<\mu_{-M}<\ldots<\mu_{-1}<0<\mu_{1}<\ldots<\mu_{M}<1
$$

with the symmetry

$$
\mu_{-m}=-\mu_{m}, \quad \text { and } \quad \alpha_{-m}=\alpha_{m}, \quad \text { for } m \in \mathcal{M}
$$

In addition, these quadrature points and weights are assumed to satisfy the quadrature conditions

$$
\begin{array}{ll}
\sum_{m=1}^{M} \mu_{m}^{2 k} \alpha_{m}=1 & \text { for } k=0 \\
\sum_{m=1}^{M} \mu_{m}^{2 k} \alpha_{m}=\frac{1}{3} & \text { for } k=1 .
\end{array}
$$

Additional conditions might also be assumed (see $[12,14,15]$ ). The conditions above are met by many quadrature sets, for instance, by the classical Gauss quadrature over $[-1,1]$, or the so-called "double Gauss" quadrature over $[-1,0] \cup[0,1]$ provided that each half interval range contains at least 2 quadrature points. The value of the exact solution $\Psi^{\epsilon}(x, \mu)$ of the steady transport equation (1.1)-(1.3) at the quadrature points $\mu_{m}$ is then formally approximated by the discrete particle densities $\psi^{\epsilon} \equiv \psi_{m}^{\epsilon}(x)$ that satisfy the so-called discrete-ordinate equation

$$
\mu_{m} \partial_{x} \psi_{m}^{\epsilon}+\sigma(x) \psi_{m}^{\epsilon}=\sigma(x) c(x) \overline{\psi^{\epsilon}}
$$


where

$$
\overline{\psi^{\epsilon}}(x)=\frac{1}{2} \sum_{1 \leq|n| \leq M} \psi_{n}^{\epsilon}(x) \alpha_{n}
$$

and with boundary conditions given by

$$
\psi_{m}^{\epsilon}\left(x_{L}\right)=f_{L, m}, \quad \psi_{-m}^{\epsilon}\left(x_{R}\right)=f_{R, m}, \quad \text { for } m>0 .
$$

The discretized boundary data $f_{L, m}$ and $f_{R, m}$ can be defined in various ways from the original boundary data $F_{L}$ and $F_{M}$, as discussed in $[12,14]$. The analysis of $(5.19,5.20)$ under the conditions $(5.17)$ and (5.18) exactly parallels that of $(1.1,1.2)$, at the expense of replacing the measure $\mathrm{d} \mu$ by its discrete analogue $\sum_{1 \leq|k| \leq M} \delta_{\mu_{k}}$. In the diffusive domain $x_{M}<x<x_{R}, \sigma(x)=\epsilon^{-1}$ while $c(x)=1-\epsilon^{2} \gamma(x)$; hence

$$
\begin{aligned}
\mu_{m} \partial_{x} \psi_{m}^{\epsilon}+\epsilon^{-1} \psi_{m}^{\epsilon} & =\epsilon^{-1}\left(1-\epsilon^{2} \gamma\right) \overline{\psi^{\epsilon}}, & & x_{M}<x<x_{R}, \\
\psi_{m}^{\epsilon}\left(x_{M}\right) & =g_{m}, & & \text { for } m>0, \\
\psi_{-m}^{\epsilon}\left(x_{R}\right) & =f_{R, m}, & & \text { for } m>0 .
\end{aligned}
$$

The boundary data $g_{m}$ will be determined later.

The diffusion approximation to the discrete-ordinate equation $(5.21)$ is (see $[12,14,22]$ )

$$
\psi_{m}^{\epsilon}=\theta^{\epsilon}-\epsilon \mu_{m} \partial_{x} \theta^{\epsilon}+O\left(\epsilon^{2}\right)
$$

where $\theta^{\epsilon}$ satisfies the diffusion equation

$$
-\frac{1}{3} \partial_{x x} \theta^{\epsilon}+\gamma \theta^{\epsilon}=0, \quad x \in\left(x_{M}, x_{R}\right)
$$

and the boundary conditions

$$
\begin{aligned}
& \theta^{\epsilon}-\left.\epsilon \lambda_{\text {disc }} \partial_{x} \theta^{\epsilon}\right|_{x=x_{M}}=\sum_{m=1}^{M} \psi_{m}^{\epsilon}\left(x_{M}\right) w_{m} \\
& \theta^{\epsilon}+\left.\epsilon \lambda_{\text {disc }} \partial_{x} \theta^{\epsilon}\right|_{x=x_{R}}=\sum_{m=1}^{M} f_{R, m} w_{m} .
\end{aligned}
$$

Obtaining the boundary conditions (5.23) can be done exactly as in the case of a continuous angular variable $\mu$ : see [14]. This involves discrete ordinate analogs of the half-space problem studied in Section 2. In particular, one obtains discrete versions of the Chandrasekhar $H$-function and of the operator $\mathcal{R}$, by replacing the measure $\mathrm{d} \mu$ with its discrete analog $\sum_{1 \leq|k| \leq M} \delta_{\mu_{k}}$ in the procedure described in Section 2. Alternately, one can follow the analysis in [6], originally done in the context of the discrete ordinate equation. We review this analysis below in its slightly modified form to be found in [14].

The extrapolated endpoint distance $\lambda_{\text {disc }}$ in (5.23) measured in mean free paths is given by (see [14])

$$
\lambda_{\text {disc }}=\sum_{m=1}^{M} \mu_{m}-\sum_{n=1}^{M-1} \frac{1}{\xi_{n+\frac{1}{2}}},
$$

where $\xi=\xi_{n+\frac{1}{2}}$ is the unique (positive, simple) root of the characteristic equation (see [6], Chap. III, eq. (6))

$$
1=\frac{1}{2} \sum_{m \in \mathcal{M}} \frac{\alpha_{m}}{1-\mu_{m} \xi},
$$


that lies in the open interval $\left(1 / \mu_{n+1}, 1 / \mu_{n}\right)$. The weights $w_{m}$ that appear in $(5.23)$ are computed in terms of the $\mu_{m}$ and the $\xi_{n+1 / 2}$ by the formula

$$
w_{m}=\prod_{n=1}^{M-1}\left(\mu_{m}-\frac{1}{\xi_{n+\frac{1}{2}}}\right) \prod_{\substack{k=1 \\ k \neq m}}^{M}\left(\frac{1}{\mu_{m}-\mu_{k}}\right)>0 .
$$

These expressions for $\lambda_{\text {disc }}$ and the $w_{m}$ are derived in [14] — see equations (A.17) and (A.18) — through a discrete boundary layer analysis. The $w_{m}$ 's are the discrete analog of Chandrasekhar's $H$-function of Definition 2.1 . Finally, we need the discrete version of the reflection operator $\mathcal{R}$. In the discrete case, the problem analogous to determining $\Gamma(0,-\mu)$ in terms of $G$ (with the notations of Lem. 2.2) reduces to Lagrange interpolation. One finds that the discrete analog of $\mathcal{R}$ is

$$
\left(\mathcal{R}_{\mathrm{disc}} g\right)_{m} \equiv(-1)^{M-1} \sum_{k=1}^{M} g_{k}\left(\prod_{j=1}^{M-1} \frac{1-\xi_{j+\frac{1}{2}} \mu_{k}}{1+\xi_{j+\frac{1}{2}} \mu_{m}} \prod_{\substack{i=1 \\ i \neq k}}^{M} \frac{\mu_{m}+\mu_{i}}{\mu_{k}-\mu_{i}}\right)
$$

This formula can be found by following the argument in Appendix A of [14], after setting $b=0$ in equation (A.1) there. Proceeding as in Section 3, we formulate the coupling algorithms:

Order $\epsilon$ coupling. First solve the discrete ordinate equation

$$
\begin{aligned}
\mu_{m} \partial_{x} \phi_{m}^{0}+\phi_{m}^{0}-c \bar{\phi}_{m}^{0} & =0, & & x \in\left(x_{L}, x_{M}\right), m \in \mathcal{M}, \\
\phi_{m}^{0}\left(x_{L}\right) & =f_{L, m}, & & 1 \leq m \leq M \\
\phi_{-m}^{0}\left(x_{M}\right) & =\mathcal{R}_{\operatorname{disc}}\left(\phi^{0}\left(x_{M}\right)\right)_{m}, & & 1 \leq m \leq M .
\end{aligned}
$$

Once this is done and the density $\phi_{0}$ has been computed, solve the diffusion problem

$$
\begin{aligned}
-\frac{1}{3} \partial_{x x} \theta^{0}+\gamma \theta^{0} & =0, \quad x \in\left(x_{M}, x_{R}\right), \\
\theta^{0}\left(x_{M}\right) & =\sum_{m=1}^{M} \phi_{m}^{0}\left(x_{M}\right) w_{m}, \\
\theta^{0}\left(x_{R}\right) & =\sum_{m=1}^{M} f_{R, m}\left(x_{M}\right) w_{m} .
\end{aligned}
$$

When keeping $M$ fixed and letting $\epsilon$ converge to zero, the solution $\psi_{m}^{\epsilon}$ is approximated by

$$
\begin{array}{ll}
\psi_{m}^{0}(x)=\phi_{m}^{0}(x), & x \in\left(x_{L}, x_{M}\right), m \in \mathcal{M}, \\
\psi_{m}^{0}(x)=\theta^{0}(x), & x \in\left(x_{L}, x_{M}\right), m \in \mathcal{M},
\end{array}
$$

to within $O(\epsilon)$.

Order $\epsilon^{\mathbf{2}}$ coupling. With $\theta^{0}$ computed as in the previous step, solve the discrete ordinate equation

$$
\begin{aligned}
\mu_{m} \partial_{x} \phi_{m}^{1}+\phi_{m}^{1}-c \bar{\phi}_{m}^{1} & =0, & & x \in\left(x_{L}, x_{M}\right), m \in \mathcal{M}, \\
\phi_{m}^{1}\left(x_{L}\right) & =0, & & 1 \leq m \leq M, \\
\phi_{-m}^{1}\left(x_{M}\right) & =\mathcal{R}_{\operatorname{disc}}\left(\phi^{1}\left(x_{M}\right)\right)_{m}+\left(\mu_{m}-\mathcal{R}_{\operatorname{disc}}(\mu)_{m}\right) \partial_{x} \theta^{0}\left(x_{M}\right), & & 1 \leq m \leq M .
\end{aligned}
$$


Once this is done $\theta^{0}, \phi^{0}$ and $\phi^{1}$ have been computed and one can solve the diffusion problem

$$
\begin{aligned}
-\frac{1}{3} \partial_{x x} \theta_{\epsilon}+\gamma \theta_{\epsilon} & =0, \quad x \in\left(x_{M}, x_{R}\right), \\
\theta_{\epsilon}\left(x_{M}\right)-\left.\epsilon \lambda_{\operatorname{disc}} \partial_{x} \theta_{\epsilon}\right|_{x=x_{M}} & =\sum_{m=1}^{M}\left(\phi_{m}^{0}+\epsilon \phi_{m}^{1}\right)\left(x_{M}\right) w_{m}, \\
\left.\theta_{\epsilon}\left(x_{R}\right)\right)+\left.\epsilon \lambda_{\text {disc }} \partial_{x} \theta_{\epsilon}\right|_{x=x_{R}} & =\sum_{m=1}^{M} f_{R, m}\left(x_{M}\right) w_{m} .
\end{aligned}
$$

When keeping $M$ fixed and letting $\epsilon$ converge to zero, the solution $\psi_{m}^{\epsilon}$ is approximated by

$$
\begin{array}{ll}
\psi_{m}^{1, \epsilon}(x)=\phi_{m}^{0}(x)+\epsilon \phi_{m}^{1}(x), & x \in\left(x_{L}, x_{M}\right), m \in \mathcal{M}, \\
\psi_{m}^{1, \epsilon}(x)=\theta_{\epsilon}^{0}(x)-\epsilon \mu_{m} \partial_{x} \theta_{\epsilon}(x), & x \in\left(x_{M}, x_{R}\right), m \in \mathcal{M},
\end{array}
$$

to within $O\left(\epsilon^{2}\right)$. The error estimates concerning the limit $\epsilon \rightarrow 0$ while keeping $M$ fixed are analogous to those in Section 4 . What happens if in addition one lets $M$ tend to $\infty$ can be analyzed by using the methods described in the present paper with those from [12].

\section{Appendix A: The transport Equation WITH REFLECTION BOUNDARY CONDITIONS}

Let $\mathcal{A}$ be a bounded, linear operator on $L^{2}([0,1], \mu \mathrm{d} \mu)$ such that

$$
\|\mathcal{A}\|_{\mathcal{L}\left(L^{2}([0,1], \mu \mathrm{d} \mu)\right.} \leq 1 .
$$

Consider the transport equation

$$
\begin{aligned}
\mu \partial_{x} \psi+\sigma(x) \psi & =\sigma(x) c(x) \bar{\psi}, & (x, \mu) \in\left(x_{L}, x_{M}\right) \times[-1,1], \\
\psi\left(x_{L}, \mu\right) & =F_{L}(\mu), & \mu \in(0,1], \\
\psi\left(x_{M},-\mu\right) & =\mathcal{A}\left(\left.\psi\left(x_{M}, \cdot\right)\right|_{(0,1]}\right)(\mu)+\phi_{M}(\mu), & \mu \in(0,1] .
\end{aligned}
$$

Proposition A.1. Assume that the measurable functions $\sigma$ and $c$ satisfy the bounds $0<\sigma_{*} \leq \sigma(x) \leq \sigma^{*}$ and $0 \leq c(x) \leq c^{*}<1$ for each $x \in\left(x_{L}, x_{R}\right)$ and some constants $\sigma_{*}, \sigma^{*}$ and $c^{*}$. For each $F_{L}$ and $\phi_{M} \in L^{2}([0,1], \mu \mathrm{d} \mu)$, there exists a unique solution $\psi$ to $(A .2)$ in $L^{2}\left(\left(x_{L}, x_{M}\right) \times[-1,1]\right)$.

Proof. We use the objects and notations from Lemma 4.1. Assume the existence of a solution $\psi \in L^{2}\left(\left(x_{L}, x_{M}\right) \times\right.$ $[-1,1])$; the transport equation implies that $\mu \partial_{x} \psi$ is in $L^{2}\left(\left(x_{L}, x_{M}\right) \times[-1,1]\right)$, so that $\psi\left(x_{M}, \mu\right)$ is a well-defined element in $L^{2}([-1,1],|\mu| \mathrm{d} \mu)$. Define $F_{M}(\mu)=\psi\left(x_{M},-\mu\right)$ for $\mu \in(0,1]$; thus the boundary condition at $x_{M}$ is equivalent to the equality

$$
F_{M}=\mathcal{A} \circ\left(\mathcal{T}_{L} F_{L}+\mathcal{T}_{M} F_{M}\right)+\phi_{M}
$$

By Lemma 4.1, $\left\|\mathcal{A} \circ \mathcal{T}_{M}\right\|_{\mathcal{L}\left(L^{2}([0,1], \mu \mathrm{d} \mu)\right.}<1$, thus $I-\mathcal{A} \circ \mathcal{T}_{M}$ is invertible in $\mathcal{L}\left(L^{2}([0,1], \mu \mathrm{d} \mu)\right.$. Hence

$$
F_{M}=\left(I-\mathcal{A} \circ \mathcal{T}_{M}\right)^{-1}\left(\mathcal{A} \circ \mathcal{T}_{L} F_{L}+\phi_{M}\right) ;
$$


this shows that $F_{M} \in L^{2}([0,1], \mu \mathrm{d} \mu)$ is uniquely defined in terms of $F_{L}$ and $\phi_{M}$, so that there is at most one solution $\psi$ of $\left(\right.$ A.2) in $L^{2}\left(\left(x_{L}, x_{M}\right) \times[-1,1]\right)$. Conversely, let $\tilde{\psi}$ be the solution of

$$
\begin{array}{rlrl}
\mu \partial_{x} \tilde{\psi}+\sigma(x) \tilde{\psi} & =\sigma(x) c(x) \bar{\psi}, & & (x, \mu) \in\left(x_{L}, x_{M}\right) \times[-1,1], \\
\tilde{\psi}\left(x_{L}, \mu\right) & =F_{L}(\mu), & \mu \in(0,1], \\
\tilde{\psi}\left(x_{M},-\mu\right) & =\left(I-\mathcal{A} \circ \mathcal{T}_{M}\right)^{-1}\left(\mathcal{A} \circ \mathcal{T}_{L} F_{L}+\phi_{M}\right)(\mu), & & \mu \in(0,1] .
\end{array}
$$

Since $F_{L}$ and $\phi_{M} \in L^{2}([0,1], \mu \mathrm{d} \mu)$, the boundary term

$$
\left(I-\mathcal{A} \circ \mathcal{T}_{M}\right)^{-1}\left(\mathcal{A} \circ \mathcal{T}_{L} F_{L}+\phi_{M}\right),
$$

also belongs to $L^{2}([0,1], \mu \mathrm{d} \mu)$ so that the above transport equation has a unique solution $\tilde{\psi} \in L^{2}\left(\left(x_{L}, x_{M}\right) \times\right.$ $[-1,1])$. But the boundary condition at $x_{M}$ implies that

$$
\tilde{\psi}\left(x_{M},-\mu\right)=\mathcal{A}\left(\left.\tilde{\psi}\left(x_{M}, \cdot\right)\right|_{(0,1]}\right)(\mu)+\phi_{M}(\mu),
$$

so that $\tilde{\psi}$ coincides with $\psi$ by the uniqueness property proved above.

\section{Appendix B: The multi-Dimensional Chandrasekhar function}

The adjoint problem

$$
\begin{aligned}
(\omega \cdot \nu) \partial_{z} v+v-\bar{v} & =0, & & z>0, \omega \in \mathbf{S}^{D-1}, \\
v(0, \omega) & =0, & & \omega \cdot \nu>0, \\
\frac{(\omega \cdot \nu) v}{} & =-1, & & z>0,
\end{aligned}
$$

has a unique solution $v \equiv v(z, \omega)$ such that

$$
(z, \omega) \mapsto v(z, \omega)-\frac{1}{K^{2}} z \text { belongs to } L^{\infty}\left(\mathbf{R}_{+} ; L^{2}\left(\mathbf{S}^{D-1},|\omega \cdot \nu| \mathrm{d} \omega\right)\right),
$$

where $K=\left(\overline{(\omega \cdot \nu)^{2}}\right)^{1 / 2}$. It is defined by the formula

$$
v(z, \omega)=v_{0}(z, \omega)+\frac{1}{K^{2}}(z-(\omega \cdot \nu))
$$

where $v_{0}$ is the unique solution in $L^{\infty}\left(\mathbf{R}_{+} ; L^{2}\left(\mathbf{S}^{D-1},|\omega \cdot \nu| \mathrm{d} \omega\right)\right)$ of the problem

$$
\begin{aligned}
(\omega \cdot \nu) \partial_{z} v_{0}+v_{0}-\bar{v}_{0} & =0, & & z>0, \omega \in \mathbf{S}^{D-1}, \\
v_{0}(0, \omega) & =\omega \cdot \nu, & & \omega \cdot \nu>0 .
\end{aligned}
$$

Let $\Gamma \in L^{\infty}\left(\mathbf{R}_{+} ; L^{2}\left(\mathbf{S}^{D-1},|\omega \cdot \nu| \mathrm{d} \omega\right)\right)$ be the unique solution of (5.11). Observe that

$$
\frac{\mathrm{d}}{\mathrm{d} z} \int_{\mathbf{S}^{D-1}}(\omega \cdot \nu) \Gamma(z, \omega) v(z, \omega) \mathrm{d} \omega=\int_{\mathbf{S}^{D-1}}(\Gamma(z, \omega)-\bar{\Gamma}(z)) v(z, \omega) \mathrm{d} \omega-\int_{\mathbf{S}^{D-1}} \Gamma(z, \omega)(v(z, \omega)-\bar{v}(z)) \mathrm{d} \omega=0 .
$$

By evaluating the quantity $\overline{(\omega \cdot \nu) \Gamma v}$ both at $z=0$ and for $z \rightarrow+\infty$, one finds that

$$
\int_{\mathbf{S}_{\nu}^{-}}(\omega \cdot \nu) G(\omega) v(0, \omega) \mathrm{d} \omega=\overline{(\omega \cdot \nu) v} \Lambda_{\nu}(G)
$$


leading to the formula

$$
\Lambda_{\nu}(G)=\int_{\mathbf{S}_{\nu}^{-}}|\omega \cdot \nu| G(\omega) v(0, \omega) \mathrm{d} \omega .
$$

This expression is analogous to the first formula in Lemma 2.2. In other words, the linear functional $\Lambda_{\nu}$ is represented on the Hilbert space $L^{2}\left(\mathbf{S}_{\nu}^{-},|\omega \cdot \nu| \mathrm{d} \omega\right)$ by the function $\frac{1}{K} H_{\nu}(\omega)$, where $H_{\nu}$ is defined by

$$
H_{\nu}(\omega)=K v(0, \omega) .
$$

Consider next the function

$$
u(z, \omega)=(\omega \cdot \nu) \Gamma(z, \omega)+\int_{0}^{z} \bar{\Gamma}(s) \mathrm{d} s ;
$$

it satisfies

$$
\begin{aligned}
-(\omega \cdot \nu) \partial_{z} u+u-\bar{u} & =0, & & z>0, \omega \in \mathbf{S}^{D-1}, \\
u(0, \omega) & =(\omega \cdot \nu) G(\omega), & & \omega \cdot \nu<0, \\
\frac{(\omega \cdot \nu) u}{} & =K^{2} \Lambda_{\nu}(G), & & z>0 .
\end{aligned}
$$

It also verifies the condition

$$
(z, \omega) \mapsto u(z, \omega)-\Lambda_{\nu}(G) z \text { belongs to } L^{\infty}\left(\mathbf{R}_{+} ; L^{2}\left(\mathbf{S}^{D-1},|\omega \cdot \nu| \mathrm{d} \omega\right)\right) .
$$

Let $w \in L^{\infty}\left(\mathbf{R}_{+} ; L^{2}\left(\mathbf{S}^{D-1},|\omega \cdot \nu| \mathrm{d} \omega\right)\right)$ be the solution of

$$
\begin{aligned}
-(\omega \cdot \nu) \partial_{z} w+w-\bar{w} & =0, & & z>0, \omega \in \mathbf{S}^{D-1} \\
w(0, \omega) & =(\omega \cdot \nu) G(\omega), & & \omega \cdot \nu<0 .
\end{aligned}
$$

It must also verify the condition

$$
\overline{(\omega \cdot \nu) w}=0 \text { for all } z>0 .
$$

Indeed, by averaging over $\mathbf{S}^{D-1}$ the equations for $w$ and $(\omega \cdot \nu) w$, one finds that

$$
\overline{(\omega \cdot \nu) w}=\text { const. and } \frac{\mathrm{d}}{\mathrm{d} z} \overline{(\omega \cdot \nu)^{2} w}=\overline{(\omega \cdot \nu) w} \text {; }
$$

one concludes by observing that $\overline{(\omega \cdot \nu)^{2} w} \in L^{\infty}\left(\mathbf{R}_{+}\right)$. Hence the function $u-w$ satisfies

$$
\begin{aligned}
-(\omega \cdot \nu) \partial_{z}(u-w)+(u-w)-\overline{(u-w)} & =0, & & z>0, \omega \in \mathbf{S}^{D-1}, \\
(u-w)(0, \omega) & =0, & & \omega \cdot \nu<0, \\
\overline{(\omega \cdot \nu)(u-w)} & =K^{2} \Lambda_{\nu}(G), & & z>0 .
\end{aligned}
$$

By uniqueness of the solution of (5.11) in $L^{\infty}\left(\mathbf{R}_{+} ; L^{2}\left(\mathbf{S}^{D-1},|\omega \cdot \nu| \mathrm{d} \omega\right)\right)$, one finds that

$$
(u-w)\left(z, \mathbf{s}_{\nu} \omega\right)=K^{2} \Lambda_{\nu}(G) v(z, \omega), \quad \text { where } \mathbf{s}_{\nu} \omega=\omega-2(\omega \cdot \nu) \nu .
$$

At $z=0$ and for $\omega \cdot \nu<0$, this relation can be recast as

$$
-(\omega \cdot \nu) \mathcal{R}_{\nu} G(\omega)-\left(\mathcal{R}_{\nu}(\omega \cdot \nu) G\right)(\omega)=H_{\nu}(\omega) \int_{\mathbf{S}_{\nu}^{-}}\left|\omega^{\prime} \cdot \nu\right| H_{\nu}\left(\omega^{\prime}\right) G\left(\omega^{\prime}\right) \mathrm{d} \omega^{\prime},
$$

which shows that $\mathcal{R}_{\nu}$ is of the form

$$
\mathcal{R}_{\nu} G(\omega)=\int_{\mathbf{S}_{\nu}^{-}} r_{\nu}\left(\omega, \omega^{\prime}\right) G\left(\omega^{\prime}\right)\left|\omega^{\prime} \cdot \nu\right| \mathrm{d} \omega^{\prime}
$$


with

$$
r_{\nu}\left(\omega, \omega^{\prime}\right)=\frac{H_{\nu}(\omega) H_{\nu}\left(\omega^{\prime}\right)}{|\omega \cdot \nu|+\left|\omega^{\prime} \cdot \nu\right|} .
$$

This expression is analogous to the second formula in Lemma 2.2. Obviously, the solution $\Gamma$ of (5.11) corresponding to $G \equiv 1$ is $\Gamma \equiv 1$; hence $\mathcal{R}_{\nu} 1=1$, which results in the nonlinear integral equation for $H_{\nu}$

$$
\frac{1}{H_{\nu}(\omega)}=\int_{\mathbf{S}_{\nu}^{-}} \frac{H_{\nu}\left(\omega^{\prime}\right)}{|\omega \cdot \nu|+\left|\omega^{\prime} \cdot \nu\right|}\left|\omega^{\prime} \cdot \nu\right| \mathrm{d} \omega^{\prime} .
$$

This shows that in fact $H_{\nu}$ depends only on $\omega \cdot \nu$ : specifically

$$
H_{\nu}(\omega)=\mathcal{H}_{D}(|\omega \cdot \nu|)
$$

and the nonlinear integral equation for $H_{\nu}$ can be recast in terms of $\mathcal{H}_{D}$ in the following manner:

$$
\frac{1}{\mathcal{H}_{D}(\mu)}=\left|\mathbf{S}^{D-2}\right| \int_{0}^{1} \frac{\mathcal{H}_{D}\left(\mu^{\prime}\right)}{\mu+\mu^{\prime}} \mu^{\prime}\left(1-\mu^{\prime 2}\right)^{\frac{D-3}{2}} \mathrm{~d} \mu^{\prime} .
$$

This integral equation is analogous to (2.6) and can be solved numerically by a relaxation method.

Acknowledgements. S. Jin's research was supported by the NSF under Grant No. DMS-0196106. D. Levermore's research was supported by the NSF under Grant No. DMS-9803753.

\section{REFERENCES}

[1] G. Bal and Y. Maday, Coupling of Transport and Diffusion Models in Linear Transport Theory. ESAIM: M2AN 36 (2002) 69-86.

[2] C. Bardos, R. Santos and R. Sentis, Diffusion approximation and computation of critical size. Trans. Amer. Math. Soc. 284 (1984) 617-649.

[3] A. Bensoussan, J.-L. Lions and G.C. Papanicolaou, Boundary layers and homogenization of transport processes. Publ. Res. Inst. Math. Sci. 15 (1979) 53-157.

[4] J.-F. Bourgat, P. Le Tallec, B. Perthame and Y. Qiu, Coupling Boltzmann and Euler equations without overlapping, in Domain decomposition methods in science and engineering (Como, 1992). Amer. Math. Soc., Providence, RI, Contemp. Math. 157 (1994) 377-398.

[5] C. Buet, S. Cordier, B. Lucquin-Desreux and S. Mancini, Diffusion limit of the Lorentz model: asymptotic preserving schemes. ESAIM: M2AN 36 (2002) 631-655.

[6] S. Chandrasekhar, Radiative Transfer. Dover, New York (1960).

[7] R. Dautray and J.L. Lions, Analyse Mathèmatique et Calcul Numérique pour les Sciences et les Techniques. Collection du Commissariat à l'Énergie Atomique: Série Scientifique, Masson, Paris (1985).

[8] P. Degond and C. Schmeiser, Kinetic boundary layers and fluid-kinetic coupling in semiconductors. Transport Theory Statist. Phys. 28 (1999) 31-55.

[9] S. Dellacherie, Kinetic fluid coupling in the field of the atomic vapor laser isotopic separation: numerical results in the case of a mono-species perfect gas, presented at the 23rd International Symposium on Rarefied Gas Dynamics, Whistler (British Columbia), July (2002).

[10] F. Golse, Applications of the Boltzmann equation within the context of upper atmosphere vehicle aerodynamics. Comput. Methods Appl. Mech. Engrg. 75 (1989) 299-316.

[11] F. Golse, Knudsen layers from a computational viewpoint. Transport Theory Statist. Phys. 21 (1992) 211-236.

$[12]$ F. Golse, S. Jin and C.D. Levermore, The convergence of numerical transfer schemes in diffusive regimes, I. The dicrete-ordinate method. SIAM J. Numer. Anal. 36 (1999) 1333-1369.

[13] M. Günther, P. Le Tallec, J.-P. Perlat and J. Struckmeier, Numerical modeling of gas flows in the transition between rarefied and continuum regimes. Numerical flow simulation I, (Marseille, 1997). Vieweg, Braunschweig, Notes Numer. Fluid Mech. 66 (1998) 222-241.

[14] S. Jin and C.D. Levermore, The discrete-ordinate method in diffusive regimes. Transport Theory Statist. Phys. 20 (1991) 413-439.

[15] S. Jin and C.D. Levermore, Fully discrete numerical transfer in diffusive regimes. Transport Theory Statist. Phys. 22 (1993) 739-791. 
[16] S. Jin, L. Pareschi and G. Toscani, Uniformly accurate diffusive relaxation schemes for multiscale transport equations. SIAM J. Numer. Anal. 38 (2000) 913-936.

[17] A. Klar, Convergence of alternating domain decomposition schemes for kinetic and aerodynamic equations. Math. Methods Appl. Sci. 18 (1995) 649-670.

[18] A. Klar, Asymptotic-induced domain decomposition methods for kinetic and drift-diffusion semiconductor equations. SIAM J. Sci. Comput. 19 (1998) 2032-2050.

[19] A. Klar, An asymptotic-induced scheme for nonstationary transport equations in the diffusive limit. SIAM J. Numer. Anal. 35 (1998) 1073-1094.

[20] A. Klar, H. Neunzert and J. Struckmeier, Transition from kinetic theory to macroscopic fluid equations: a problem for domain decomposition and a source for new algorithm. Transport Theory Statist. Phys. 29 (2000) 93-106.

[21] A. Klar and N. Siedow, Boundary layers and domain decomposition for radiative heat transfer and diffusion equations: applications to glass manufacturing process. European J. Appl. Math. 9 (1998) 351-372.

[22] E.W. Larsen, J.E. Morel and W.F. Miller, Jr., Asymptotic solutions of numerical transport problems in optically thick, diffusive regimes. J. Comput. Phys. 69 (1987) 283-324.

[23] J. Lehner and G.M. Wing, On the spectrum of an unsymmetric operator arising in the transport theory of neutrons. Comm. Pure Appl. Math. 8 (1955) 217-234.

[24] P. Le Tallec and F. Mallinger, Coupling Boltzmann and Navier-Stokes equations by half fluxes. J. Comput. Phys. 136 (1997) $51-67$.

[25] P. Le Tallec and M. Tidriri, Convergence analysis of domain decomposition algorithms with full overlapping for the advectiondiffusion problems. Math. Comp. 68 (1999) 585-606.

[26] M. Tidriri, New models for the solution of intermediate regimes in transport theory and radiative transfer: existence theory, positivity, asymptotic analysis, and approximations. J. Statist. Phys. 104 (2001) 291-325.

[27] N. Wiener and E. Hopf, Über eine Klasse singulärer Integralgleichungen, Sitzber. Preuss. Akad. Wiss., Sitzung der phys.-math. Klasse, Berlin (1931) 696-706.

To access this journal online: www.edpsciences.org 